The online version of this article is published within an Open Access environment subject to the conditions of the Creative Commons Attribution-NonCommercial-ShareAlike licence $<$ http://creativecommons.org/licenses/by-nc-sa/3.0/>. The written permission of Cambridge University Press must be obtained for commercial re-use.

doi:10.1017/jfm.2013.1

\title{
Can barotropic tide-eddy interactions excite internal waves?
}

\author{
M.-P. Lelong ${ }^{1,} \dagger$ and E. Kunze ${ }^{2}$ \\ ${ }^{1}$ NorthWest Research Associates, Seattle, WA 98009, USA \\ ${ }^{2}$ Applied Physics Laboratory, University of Washington, Seattle, WA 98195, USA \\ (Received 21 September 2011; revised 5 October 2012; accepted 1 January 2013; \\ first published online 13 March 2013)
}

The interaction of barotropic tidal currents and baroclinic geostrophic eddies is considered theoretically and numerically to determine whether energy can be transferred to an internal wave field by this process. The eddy field evolves independently of the tide, suggesting that it acts catalytically in facilitating energy transfer from the barotropic tide to the internal wave field, without exchanging energy with the other flow components. The interaction is identically zero and no waves are generated when the barotropic tidal current is horizontally uniform. Optimal internal wave generation occurs when the scales of tide and eddy fields satisfy resonant conditions. The most efficient generation is found if the tidal current horizontal scale is comparable to that of the eddies, with a weak maximum when the scales differ by a factor of two. Thus, this process is not an effective mechanism for internal wave excitation in the deep ocean, where tidal current scales are much larger than those of eddies, but it may provide an additional source of internal waves in coastal areas where horizontal modulation of the tide by topography can be significant.

Key words: baroclinic flows, internal waves, ocean processes

\section{Introduction}

The motivation for this study comes from surface-drifter observations in the Western Boundary Current of the subpolar North Pacific, which showed intense near-inertial/diurnal frequency motions trapped within anticyclonic warm-core rings (Rogachev et al. 1992, 1996; Rogachev \& Carmack 2002). The authors speculated that the lower bound of the internal wave frequency band had been locally decreased by the rings' negative vorticity (Kunze 1985), trapping the near-inertial waves within the rings. The proximity of the near-inertial and near-diurnal frequencies led them to speculate that the waves might have been generated by a diurnal tide-eddy interaction. However, given their limited data, they could not rule out the role of wind forcing or sub-inertial instability. The evidence for the suspected role of the barotropic tide was, by and large, circumstantial and not substantiated by any theoretical arguments. Nor does this mechanism appear to have been considered rigorously in previous work. 
The objective of this paper is to examine this overlooked mechanism for internal tide generation theoretically and numerically under mid-latitude conditions.

The best-established mechanism for the generation of linear internal waves by the surface tide in the ocean is through the interaction of surface tidal currents with topography (Cox \& Sandstrom 1962; Wunsch 1975; Simmons, Hallberg \& Arbic 2004; Garrett \& Kunze 2007). Bell (1975) developed a complete theory for the case of small-amplitude (topographic height $h \ll$ bottom depth $H$ ), gently sloped (bottom slope $\alpha<$ ray slope $s$ ) two-dimensional (2D) topography $h(x, y)$, including the case of finite excursions relative to the topographic length scale. This approach has been extended to one-dimensional (1D) cases where $\alpha / s \rightarrow 1$ (Balmforth, Ierley \& Young 2002; Llewellyn Smith \& Young 2002; St Laurent \& Garrett 2002). Thorpe (1992) and MacCready \& Pawlak (2001) examined the case of bottom roughness on a slope. The case of finite amplitude and finite slope has proven more challenging, though topographic features of these characteristics are clearly important in transferring energy from barotropic to baroclinic tides (Morozov 1995; Ray \& Mitchum 1997; Egbert \& Ray 2001). Insight has largely been gained by examining idealized 1D topographies (Khatiwala 2003; Llewellyn Smith \& Young 2003; St Laurent et al. 2003; Petrelis, Llewellyn Smith \& Young 2006), or from observations (e.g. Pingree, Mardell \& New 1986; Pingree \& New 1989; Althaus, Kunze \& Sanford 2003; Rudnick et al. 2003; Nash et al. 2006; Lee et al. 2006). Generation by general 2D topography has required numerical simulation (Holloway \& Merrifield 1999; Merrifield \& Holloway 2002; Simmons et al. 2004). A monograph (Vlasenko, Stashchuk \& Hutter 2005) and recent review (Garrett \& Kunze 2007) discuss mostly theoretical aspects of tide-topography interaction.

Here, we consider the interaction of an oscillating tidal current with a baroclinic geostrophic eddy field. The geostrophic eddy field time scale is an advective time scale, much longer than the tidal period. If the characteristic length scales of the eddy field and tide combine to match those of an internal wave of tidal frequency, a resonant interaction occurs, similar to the wave-wave triad resonant interactions that transfer energy efficiently between internal waves (McComas \& Bretherton 1977).

The general mathematical framework for delineating eddy motions, barotropic and internal tides is introduced in the following section. In $\S 3$, we examine a simple prototype flow and establish conditions under which resonance between the eddy field and barotropic tide can occur. Section 4 presents numerical simulations designed to validate and extend the analytical solutions. Discussion of our results and concluding remarks are given in $\S 5$.

\section{Problem definition}

\subsection{Wave-triad interactions}

The envisioned interaction between a barotropic tide and an eddy field bears similarities with internal wave-triad interactions (McComas \& Bretherton 1977; Müller et al. 1986) and with the resonant interaction between wind-forced, nearinertial motions and a turbulent mesoscale eddy field (Danioux \& Klein 2008). To illustrate, we consider a barotropic semidiurnal tide of frequency $\omega_{0}$ and wavevector $\kappa_{0}=\left\{0, l_{0}, 0\right\}$ and an eddy field with characteristic wavevector $\kappa_{1}=\left\{k_{1}, l_{1}, m_{1}\right\}$ and time scale $1 / k_{1} U \gg 2 \pi / \omega_{0}$, where $U$ is an eddy velocity scale. We assume that the tide does not change appreciably in the zonal direction, but we allow for meridional modulation due, for example, to topographic variations. The coordinate system is Cartesian, with $\left\{k_{i}, l_{i}, m_{i}\right\}$ denoting the two horizontal wavenumbers and $m_{i}$ the vertical 
wavenumber. Typical eddy time scales are much longer than the tidal period and the nonlinear quadratic interaction of the tide and eddy components therefore excites motions with functional form proportional to

$$
\mathrm{e}^{\mathrm{i}\left[k_{1} x \pm\left(l_{0} \pm l_{1}\right) y \pm m_{1} z \pm \omega_{0} t\right]} .
$$

Under most conditions, the interaction is weak. However, if the scales of the barotropic tide and eddy field are such that resonance conditions

$$
\begin{aligned}
\boldsymbol{\kappa}_{0} \pm \boldsymbol{\kappa}_{1} & =\boldsymbol{\kappa}_{2}, \\
\omega_{0} & =\omega\left(\boldsymbol{\kappa}_{2}\right)
\end{aligned}
$$

are satisfied for either the ' + ' or '-' relations, then a stronger interaction is possible, exciting a baroclinic tide, i.e. an inertia-gravity wave (IGW) of frequency $\omega_{2}=\omega\left(\boldsymbol{\kappa}_{2}\right)$ and wavenumber $\kappa_{2}=\left\{k_{2}, l_{2}, m_{2}\right\}=\left\{k_{1}, l_{0} \pm l_{1}, m_{1}\right\}$, where

$$
\omega\left(\kappa_{2}\right)=\sqrt{\frac{N^{2}\left(k_{2}^{2}+l_{2}^{2}\right)+f^{2} m_{2}^{2}}{k_{2}^{2}+l_{2}^{2}+m_{2}^{2}}}
$$

is the dispersion relation for an IGW with wavevector $\left\{k_{2}, l_{2}, m_{2}\right\}$ propagating in a stratified, rotating fluid; and $N$ and $f$ denote buoyancy and Coriolis frequencies, respectively. The resonant triad comprises barotropic tide, eddy field and IGW components with characteristic scales as described above. Because the time scale over which the eddy field evolves is much longer than the tidal period in our case, $\omega_{2} \approx \omega_{0}$. The relative locations of the barotropic tide, eddy and baroclinic tide components in wavenumber-frequency space are illustrated schematically in figure 1.

\subsection{Mathematical formulation}

Model equations are the $f$-plane Boussinesq equations,

$$
\begin{aligned}
\frac{\partial \boldsymbol{u}_{\boldsymbol{h}}}{\partial t}+\left(\boldsymbol{u}_{\boldsymbol{h}} \cdot \nabla_{\boldsymbol{h}}\right) \boldsymbol{u}_{\boldsymbol{h}}+w \frac{\partial \boldsymbol{u}_{\boldsymbol{h}}}{\partial z}+\frac{1}{\rho_{0}} \nabla_{\boldsymbol{h}} p+f \hat{\boldsymbol{e}}_{3} \times \boldsymbol{u}_{\boldsymbol{h}} & =\hat{\boldsymbol{e}}_{1} F(y, t), \\
\frac{\partial w}{\partial t}+\left(\boldsymbol{u}_{\boldsymbol{h}} \cdot \nabla_{\boldsymbol{h}}\right) w+w \frac{\partial w}{\partial z}+\frac{1}{\rho_{0}} \frac{\partial p}{\partial z}-b & =0 \\
\frac{\partial b}{\partial t}+\left(\boldsymbol{u}_{\boldsymbol{h}} \cdot \nabla_{\boldsymbol{h}}\right) b+w \frac{\partial b}{\partial z}+N^{2} w & =0 \\
\nabla_{\boldsymbol{h}} \cdot \boldsymbol{u}_{\boldsymbol{h}}+\frac{\partial w}{\partial z} & =0
\end{aligned}
$$

where $\boldsymbol{u}_{h}=\{u, v\}$ is the horizontal velocity, $w$ the vertical velocity, $b=-g \rho / \rho_{0}$ the buoyancy and $p$ the pressure. The buoyancy frequency $N=\sqrt{-\left(g / \rho_{0}\right)(\mathrm{d} \overline{\rho(z)} / \mathrm{d} z)}$ is defined in terms of the mean density profile $\overline{\rho(z)}$ and is taken to be constant. Vectors $\hat{\boldsymbol{e}}_{1}$ and $\hat{\boldsymbol{e}}_{3}$ are unit vectors in the $x$ and $z$ directions. Boundary conditions are periodic in $x$ and $y$ and rigid-lid free-slip in $z$.

The effect of the barotropic tide is incorporated as a time-dependent forcing term

$$
F(y, t)=U(y) \frac{\omega_{0}^{2}-f^{2}}{\omega_{0}} \cos \omega_{0} t
$$

on the right-hand side of the $u$ momentum equation. This forcing represents a parametrization of the variation of sea surface slope with the tidal cycle and is chosen 

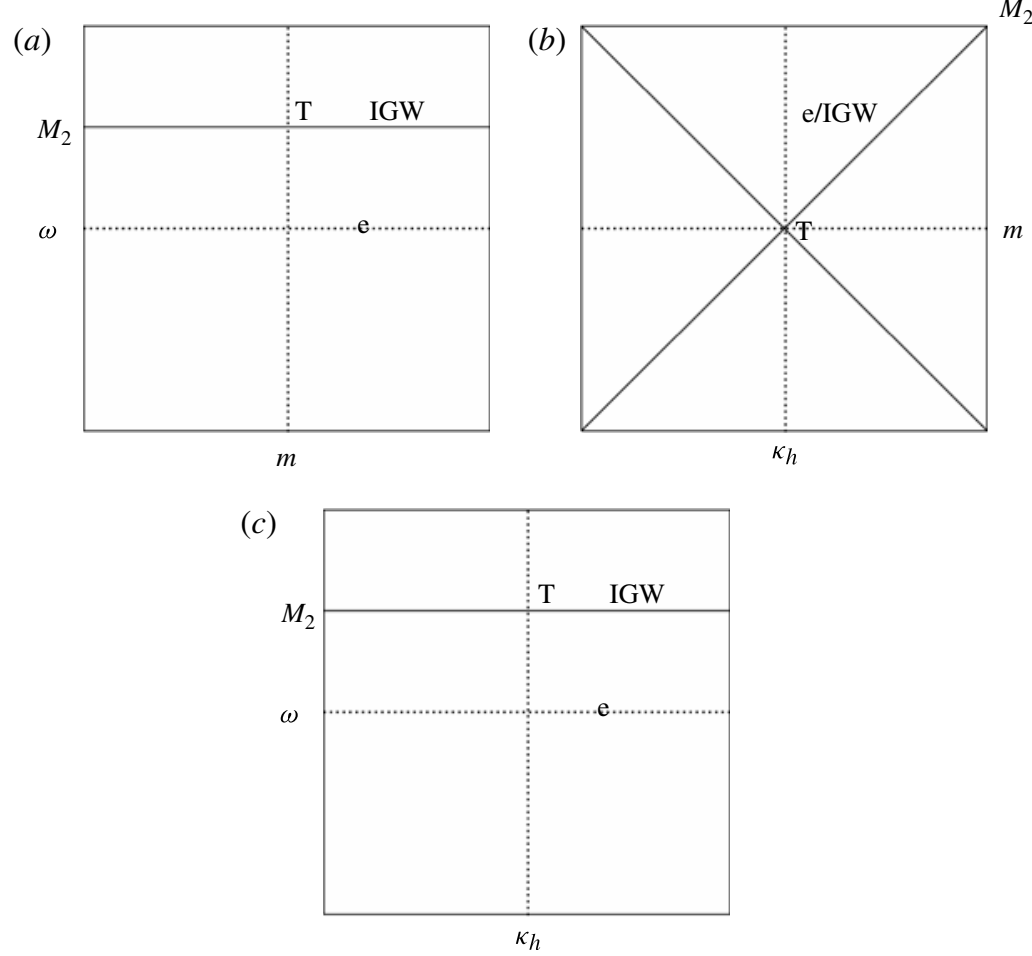

FIGURE 1. Barotropic tide (T), eddy field (e) and baroclinic tide (IGW) locations in $(a)$ vertical wavenumber-frequency space $(m, \omega),(b)$ horizontal wavenumber-vertical wavenumber space $\left(\kappa_{h}, m\right)$ and $(c)$ horizontal wavenumber-frequency space $\left(\kappa_{h}, \omega\right)$. The origin of each panel is at the centre and the dotted vertical and horizontal lines represent axes; $M_{2}$ denotes the semidiurnal tide.

to elicit a response of the form $u=U(y) \sin \omega_{0} t$, where $\omega_{0}$ denotes the tidal frequency. The eddy field is introduced as an initial condition in geostrophic and hydrostatic balance.

\subsection{Scaling}

The scaling is based on the requirements that:

(a) Coriolis and pressure-gradient terms be of the same order and present in the lowest-order eddy field equations,

$$
[P]=\rho_{0} f U L
$$

(b) buoyancy and pressure fields be in hydrostatic balance, leading to

$$
[B]=\frac{[P]}{\rho_{0} H}=\frac{f U}{\delta},
$$

where aspect ratio $\delta=H / L=W / U$.

Here, $L$ and $H$ are horizontal and vertical length scales of the eddy field, and $U$ and $W$ horizontal and vertical velocity scales of the eddy field. The Rossby number $\epsilon$, which 
represents the ratio of inertial to advective time scales

$$
\epsilon \equiv \frac{U}{f L},
$$

is introduced as a small parameter.

We make the additional reasonable assumptions that $H \sim \epsilon L$ and $W \sim \epsilon U$, which sets $\delta=O(\epsilon)$. This is justified, given typical oceanic values for $f / N$ of $O\left(10^{-2}\right)$, of the same order as $\epsilon$ based on a mid-latitude $f=10^{-4} \mathrm{~s}^{-1}$, a geostrophic eddy velocity scale $U$ of $O\left(0.1 \mathrm{~m} \mathrm{~s}^{-1}\right)$ and length scale $L$ of $O\left(10^{2} \mathrm{~km}\right)$. This implies that the Burger number $B u=(N H / f L) \sim O(1)$. With this scaling and a time scale $T_{0}=1 / f$, the non-dimensional equations are

$$
\begin{aligned}
\frac{\partial u^{*}}{\partial t^{*}}+\epsilon \boldsymbol{u}_{h}^{*} \cdot \nabla_{h}^{*} u^{*}+\epsilon w^{*} \frac{\partial u^{*}}{\partial z^{*}}+\frac{\partial p^{*}}{\partial x^{*}}-v^{*} & =U^{*}(y) \frac{\omega_{0}^{* 2}-1}{\omega_{0}^{*}} \cos \omega_{0}^{*} t^{*}, \\
\frac{\partial v^{*}}{\partial t^{*}}+\epsilon \boldsymbol{u}_{h}^{*} \cdot \nabla_{h}^{*} v^{*}+\epsilon w^{*} \frac{\partial v^{*}}{\partial z^{*}}+\frac{\partial p^{*}}{\partial y^{*}}+u^{*} & =0, \\
\epsilon^{2} \frac{\partial w^{*}}{\partial t^{*}}+\epsilon^{3} \boldsymbol{u}_{h}^{*} \cdot \nabla_{h}^{*} w^{*}+\epsilon^{3} w^{*} \frac{\partial w^{*}}{\partial z^{*}}+\frac{\partial p^{*}}{\partial z^{*}}-b^{*} & =0, \\
\frac{\partial b^{*}}{\partial t^{*}}+w^{*}+\epsilon \boldsymbol{u}_{h}^{*} \cdot \nabla_{h}^{*} b^{*}+\epsilon w^{*} \frac{\partial b^{*}}{\partial z^{*}} & =0, \\
\nabla_{h}^{*} \cdot \boldsymbol{u}_{h}^{*}+\frac{\partial w^{*}}{\partial z^{*}} & =0 .
\end{aligned}
$$

Equations $(2.9 a-e)$, along with suitable initial and boundary conditions, provide the starting point for delineation of the barotropic tide, eddy field and IGW fields. From now on, all variables will be non-dimensional but asterisks will be omitted for simplicity.

\subsection{Multiple-scale formalism}

The problem is formulated in terms of two time scales, a fast time scale $T_{0}=1 / f$ characterizing the barotropic tide and IGWs, and a slow advective time scale $T_{1}=L / U$ describing the evolution of the eddy field. The Rossby number $\epsilon$ is given by the ratio of these two time scales. The choice of fast time scales is not unique: one could also use the tidal period. However, our definition of $T_{0}$ results in simpler equations since inertial motions are natural modes of the rotating Boussinesq equations.

In terms of these two variables, the partial time derivative becomes

$$
\frac{\partial}{\partial t}=\frac{\partial}{\partial t_{0}}+\epsilon \frac{\partial}{\partial t_{1}}
$$

where $t_{0}=t / T_{0}$ and $t_{1}=t / T_{1}$.

\section{Weakly nonlinear theory}

At this point, each flow variable $G=\{u, v, w, \rho, p\}$ is expanded in powers of the Rossby number $\epsilon$, i.e.

$$
G\left(\boldsymbol{x} ; t_{0}, t_{1}\right)=G^{(0)}\left(\boldsymbol{x} ; t_{0}, t_{1}\right)+\epsilon G^{(1)}\left(\boldsymbol{x} ; t_{0}, t_{1}\right)+O\left(\epsilon^{2}\right) .
$$

This power series is unique provided that each $G^{(i)}$ remains $O(1)$ for all time (Kevorkian \& Cole 1981). Strictly speaking, this representation is only exact for 
times less than $1 / \epsilon$. For longer times, additional slow-time variables $\left(\sim \epsilon^{2} t, \epsilon^{3} t, \ldots\right)$ need to be introduced.

The next step consists of delineating eddy, tidal and internal wave components, and deriving the equations that govern their respective time evolution.

\subsection{Eddy, tide and wave definitions}

The eddy field is defined as the temporal average of the flow over the fast time scale (Reznik, Zeitlin \& Ben Jelloul 2001; Zeitlin, Reznik \& Ben Jelloul 2003). It represents the slow component at each order in $\epsilon$. For velocity,

$$
\boldsymbol{u}_{e}^{(i)}\left(\boldsymbol{x}, t_{1}\right) \equiv\left\langle\boldsymbol{u}^{(i)}\left(\boldsymbol{x} ; t_{0}, t_{1}\right)\right\rangle=\lim _{T \rightarrow \infty} \frac{1}{T} \int_{t}^{t+T} \boldsymbol{u}^{(i)}\left(\boldsymbol{x} ; \tau_{0}, t_{1}\right) \mathrm{d} \tau_{0},
$$

where $T_{0} \sim T \ll T_{1}$. Generally, the inclusion of a limit as $T \rightarrow \infty$ is needed in (3.2) to eliminate term contributions from the upper limit of integration. Other eddy flow variables are similarly defined.

The fast component, denoted by the subscript ' $f$ ', is

$$
\boldsymbol{u}_{f}^{(i)}\left(\boldsymbol{x} ; t_{0}, t_{1}\right)=\boldsymbol{u}^{(i)}\left(\boldsymbol{x} ; t_{0}, t_{1}\right)-\boldsymbol{u}_{e}^{(i)}\left(x, t_{1}\right) .
$$

This representation is unique since, by definition, fast variables have zero mean over the fast time scale,

$$
\frac{1}{T} \int_{0}^{T} \boldsymbol{u}_{f}^{(i)}\left(\boldsymbol{x} ; t_{0}, t_{1}\right) \mathrm{d} t_{0}=0
$$

The fast component is further split into a depth-mean (barotropic) part for the tide,

$$
\boldsymbol{u}_{T}^{(i)}\left(x, y ; t_{0}, t_{1}\right) \equiv \overline{\boldsymbol{u}_{f}^{(i)}\left(\boldsymbol{x} ; t_{0}, t_{1}\right)}=\frac{1}{H} \int_{-H}^{0} \boldsymbol{u}_{f}^{(i)}\left(x, y, z ; t_{0}, t_{1}\right) \mathrm{d} z,
$$

and a baroclinic perturbation about this average, representing the internal wave field,

$$
\boldsymbol{u}_{w}^{(i)}=\boldsymbol{u}_{f}^{(i)}-\boldsymbol{u}_{T}^{(i)}
$$

where

$$
\frac{1}{H} \int_{-H}^{0} \boldsymbol{u}_{w}^{(i)} \mathrm{d} z=0
$$

ensures the uniqueness of the second decomposition.

Equations governing the eddy flow are derived by applying the temporal average (defined by (3.2)) to (2.9) at each order in $\epsilon$. Fast-flow equations are obtained by subtracting temporally averaged equations from the full equations (2.9). The barotropic tide satisfies the depth-averaged fast-flow equations and the internal wave field, the difference of fast-flow equations and depth-averaged equations.

Initial conditions, defining the eddy field at $t=0$, are functions of $x, y$ and $z$, satisfy thermal-wind balance and do not depend on $\epsilon$. The tide is zero initially and is introduced through a forcing term in the momentum equations. To simplify the analysis, spatial dependence of the tidal forcing is chosen to be sinusoidal.

\subsection{Lowest order}

\subsubsection{Eddy field}

At lowest order, eddy field equations are

$$
\nabla p_{e}^{(0)}+\hat{\boldsymbol{\imath}}_{3} \times \boldsymbol{u}_{e}^{(0)}+b_{e}^{(0)} \hat{\boldsymbol{\imath}}_{3}=0,
$$




$$
\begin{aligned}
w_{e}^{(0)} & =0, \\
\nabla \cdot \boldsymbol{u}_{e}^{(0)} & =0 .
\end{aligned}
$$

Lowest-order eddy solutions replicate the spatial structure of the initial conditions, left unspecified for now. Their slow-time behaviour remains undetermined at this order.

\subsubsection{Barotropic tide}

At lowest order, the barotropic tide obeys

$$
\begin{aligned}
\frac{\partial u_{T}^{(0)}}{\partial t_{0}}-v_{T}^{(0)} & =U_{T} \frac{\left(\omega_{0}^{2}-1\right)}{\omega_{0}} \cos l_{0} y \cos \omega_{0} t_{0}, \\
\frac{\partial v_{T}^{(0)}}{\partial t_{0}}+u_{T}^{(0)} & =-\frac{\partial p_{T}^{(0)}}{\partial y}, \\
p_{T}^{(0)}(0)-p_{T}^{(0)}(-H) & =b_{T}^{(0)}, \\
\frac{\partial b_{T}^{(0)}}{\partial t_{0}}+w_{T}^{(0)} & =0, \\
\nabla_{h} \cdot \boldsymbol{u}_{T}^{(0)} & =0,
\end{aligned}
$$

with $u_{T}^{(0)}(\boldsymbol{x}, t=0)=v_{T}^{(0)}(\boldsymbol{x}, t=0)=w_{T}^{(0)}(\boldsymbol{x}, t=0)=b_{T}^{(0)}(\boldsymbol{x}, t=0)$. Since $w(z=0)=$ $w(z=-H)=0$, we have $w_{T}^{(0)} \equiv 0, b_{T}^{(0)} \equiv 0$ and $p_{T}^{(0)}\left(x, y, 0 ; t_{0}, t_{1}\right)=p_{T}^{(0)}\left(x, y,-H ; t_{0}, t_{1}\right)$.

For the horizontal tidal velocity, two cases must be distinguished, depending on whether the tidal forcing is a function of $y$ or not.

(i) If there is no $y$ dependence $\left(l_{0}=0\right)$, then (3.9) can be combined into a single equation,

$$
\frac{\partial^{2} u_{T}^{(0)}}{\partial t_{0}^{2}}+u_{T}^{(0)}=-U_{T}\left(\omega_{0}^{2}-1\right) \sin \omega_{0} t_{0},
$$

with initial conditions

$$
u_{T}^{(0)}(0)=0
$$

and

$$
\left.\frac{\partial u_{T}^{(0)}}{\partial t_{0}}\right|_{t=0}=U_{T} \frac{\left(\omega_{0}^{2}-1\right)}{\omega_{0}},
$$

leading to linear combinations of tidal frequency and inertial wave responses,

$$
\begin{aligned}
& u_{T}^{(0)}=U_{T}\left(\sin \left[\omega_{0} t_{0}+\phi\left(t_{1}\right)\right]-\frac{1}{\omega_{0}} \sin t_{0}\right), \\
& v_{T}^{(0)}=-U_{T} \frac{1}{\omega_{0}}\left(\cos \left[\omega_{0} t_{0}+\phi\left(t_{1}\right)\right]-\cos t_{0}\right) .
\end{aligned}
$$

Even though the tidal forcing amplitude $U_{T}$ is constant, the tidal solution may still undergo time modulation through a slowly varying phase $\phi\left(t_{1}\right)$, where $\phi(0)=0$. Equivalently, the slow-time variation can be taken into account by defining a complex amplitude $u_{B T}^{(0)}=U_{T} \mathrm{e}^{\mathrm{i} \phi\left(t_{1}\right)}$. The $\sin t_{0}$ and $\cos t_{0}$ terms are homogeneous solutions of the linear equations of motion and represent free oscillations of the fluid at the natural (Coriolis) frequency of the system. When Coriolis and tidal frequencies coincide, the 
forcing term in (3.9a) vanishes, resulting in a trivial solution. The strongest forcing occurs when $\omega_{0}$ is much greater than the Coriolis frequency $\left(\omega_{0} \gg 1\right)$. In this case, the tidal response becomes purely zonal, with $v_{T}^{(0)} \rightarrow 0$.

(ii) If $l_{0} \neq 0$, then $v_{T}^{(0)} \equiv 0$ in order to satisfy continuity, and (3.9) yields

$$
\begin{aligned}
& u_{T}^{(0)}=U_{T} \frac{\left(\omega_{0}^{2}-1\right)}{\omega_{0}^{2}} \cos l_{0} y \sin \omega_{0} t_{0}, \\
& p_{T}^{(0)}=-\int u_{T}^{(0)} \mathrm{d} y .
\end{aligned}
$$

In this case, the tidal solution does not include inertial oscillations.

\subsubsection{Internal inertia-gravity wave field}

Inertia-gravity waves satisfy

$$
\begin{aligned}
\frac{\partial \boldsymbol{u}_{h_{w}}^{(0)}}{\partial t_{0}}+\hat{\boldsymbol{\imath}}_{3} \times \boldsymbol{u}_{w}^{(0)} & =-\nabla_{h} p_{w}^{(0)}, \\
\frac{\partial p_{w}^{(0)}}{\partial z} & =b_{w}^{(0)}, \\
\frac{\partial b_{w}^{(0)}}{\partial t_{0}}+w_{w}^{(0)} & =0, \\
\frac{\partial u_{w}^{(0)}}{\partial x}+\frac{\partial v_{w}^{(0)}}{\partial y}+\frac{\partial w_{w}^{(0)}}{\partial z} & =0,
\end{aligned}
$$

where $\boldsymbol{u}_{h_{w}}^{(0)}=\left\{u_{w}^{(0)}, v_{w}^{(0)}\right\}$. Equations $(3.15 a-d)$ can be reduced to a wave equation,

$$
\frac{\partial^{2}}{\partial t_{0}^{2}}\left(\frac{\partial^{2} w_{w}^{(0)}}{\partial z^{2}}\right)+\nabla^{2} w_{w}^{(0)}=0,
$$

with homogeneous initial conditions. In the absence of external forcing, $w_{w}^{(0)}\left(\boldsymbol{x} ; t_{0}, t_{1}\right)=u_{w}^{(0)}\left(\boldsymbol{x} ; t_{0}, t_{1}\right)=v_{w}^{(0)}\left(\boldsymbol{x} ; t_{0}, t_{1}\right)=b_{w}^{(0)}\left(\boldsymbol{x} ; t_{0}, t_{1}\right) \equiv 0$. Therefore, no waves are excited at lowest order.

\subsection{The $O(\epsilon)$ system}

\subsubsection{Eddy field}

At $O(\epsilon)$, the eddy equations are

$$
\begin{aligned}
\nabla p_{e}^{(1)}+\hat{\boldsymbol{\imath}}_{3} \times \boldsymbol{u}_{e}^{(1)}+b_{e}^{(1)} \hat{\boldsymbol{\imath}}_{3} & =-\frac{\partial \boldsymbol{u}_{e}^{(0)}}{\partial t_{1}}-\left\langle\boldsymbol{u}^{(0)} \cdot \nabla \boldsymbol{u}^{(0)}\right\rangle, \\
w_{e}^{(1)} & =-\frac{\partial b_{e}^{(0)}}{\partial t_{1}}-\left\langle\boldsymbol{u}_{e}^{(0)} \cdot \nabla b_{e}^{(0)}\right\rangle, \\
\nabla \cdot \boldsymbol{u}_{e}^{(1)} & =0
\end{aligned}
$$

where \langle\rangle denotes temporal averaging. The initial conditions are homogeneous. Taking the vertical component of the curl of $(3.17 a)$, the $z$ derivative of $(3.17 b)$ and combining the resulting equations yields an equation describing the slow-time behaviour of the $O(1)$ eddy solution. The last term in (3.17a) contains only contributions from eddy-eddy interactions since the fast-fast interactions, which, at 
this order, only involve the tide, are identically zero. In terms of a stream function $\psi^{(0)}, u_{e}^{(0)}=-\psi_{y}^{(0)}, v_{e}^{(0)}=\psi_{x}^{(0)}$ and $b_{e}^{(0)}=-\psi_{z}^{(0)}$, the slow evolution is governed by

$$
\frac{\partial \Pi^{(0)}}{\partial t_{1}}+\frac{\partial\left(\psi^{(0)}, \Pi^{(0)}\right)}{\partial(x, y)}=0,
$$

the conservation equation for the lowest-order potential vorticity $\Pi^{(0)}=\nabla^{2} \psi^{(0)}$, where $\nabla^{2}$ is the three-dimensional Laplacian. An important result is that the eddy solution evolves on the slow time independently of the tide. This type of behaviour could have been anticipated since it is characteristic of interactions between flow components with disparate time scales (e.g. Lelong \& Riley 1991).

\subsubsection{Barotropic tide}

The barotropic tide equations are

$$
\begin{aligned}
\frac{\partial \boldsymbol{u}_{T}^{(1)}}{\partial t_{0}}+\hat{\boldsymbol{\imath}}_{3} \times \boldsymbol{u}_{T}^{(1)}+\nabla_{h} p_{T}^{(1)} & =-\frac{\partial \boldsymbol{u}_{T}^{(0)}}{\partial t_{1}}-\overline{\boldsymbol{u}_{e}^{(0)} \cdot \nabla_{h} \boldsymbol{u}_{T}^{(0)}}-\overline{\boldsymbol{u}_{T}^{(0)} \cdot \nabla_{h} \boldsymbol{u}_{e}^{(0)}} \\
\frac{1}{H}\left(p_{T}^{(1)}(z=0)-p_{T}^{(1)}(z=-H)\right) & =b_{T}^{(1)} \\
\frac{\partial b_{T}^{(1)}}{\partial t_{0}}+w_{T}^{(1)} & =-\frac{\partial b_{T}^{(0)}}{\partial t_{1}}-\overline{\boldsymbol{u}_{T}^{(0)} \cdot \nabla_{h} b_{e}^{(0)}} \\
\frac{\partial u_{T}^{(1)}}{\partial x}+\frac{\partial v_{T}^{(1)}}{\partial y} & =0
\end{aligned}
$$

where, as in the $O(1)$ solutions, $w_{T}^{(1)} \equiv 0$, owing to the vertical boundary conditions. The overbar denotes vertical averaging. Tide-tide interactions are identically zero. The slow-time evolution of $u_{T}^{(0)}$ is governed by a kinetic energy equation,

$$
\frac{1}{2} \frac{\partial}{\partial t_{1}}\left\|u_{T}^{(0)}\right\|^{2}=u_{T}^{(0)} v_{T}^{(1)}-u_{T}^{(0)} \frac{\partial u_{T}^{(1)}}{\partial t_{0}}+u_{T}^{(0)} M\left(\boldsymbol{u}_{e}^{(0)}, u_{T}^{(0)}\right),
$$

and $M$ is given by the last two terms of (3.19a). Equation (3.20) includes eddy-tide interaction terms, in contrast to (3.18), which is decoupled from tidal flow. Equation (3.20) is a mixed-order equation and cannot be solved without explicitly solving for $\boldsymbol{u}_{T}^{(1)}$, a consequence of the absence of wave excitation at lowest order. However, the behaviour of the potential vorticity is strongly reminiscent of situations where the eddy field plays a catalytic role in transferring energy out of the fast component but does not exchange energy with it (e.g. Lelong \& Riley 1991; Waite \& Bartello 2004).

\subsubsection{Internal inertia-gravity waves}

The baroclinic equations are

$$
\begin{aligned}
& \frac{\partial \boldsymbol{u}_{w}^{(1)}}{\partial t_{0}}+\hat{\boldsymbol{\imath}}_{3} \times \boldsymbol{u}_{w}^{(1)}+\nabla_{h} p_{w}^{(1)} \\
& =-\frac{\partial \boldsymbol{u}_{w}^{(0)}}{\partial t_{1}}-\boldsymbol{u}_{e}^{(0)} \cdot \nabla_{h} \boldsymbol{u}_{T}^{(0)}+\overline{\boldsymbol{u}_{e}^{(0)} \cdot \nabla_{h} \boldsymbol{u}_{T}^{(0)}}-\boldsymbol{u}_{T}^{(0)} \cdot \nabla_{h} \boldsymbol{u}_{e}^{(0)}+\overline{\boldsymbol{u}_{T}^{(0)} \cdot \nabla_{h} \boldsymbol{u}_{e}^{(0)}} \\
& \quad-\boldsymbol{u}_{T}^{(0)} \cdot \nabla_{h} \boldsymbol{u}_{T}^{(0)}+\overline{\boldsymbol{u}_{T}^{(0)} \cdot \nabla_{h} \boldsymbol{u}_{T}^{(0)}}+\left\langle\boldsymbol{u}_{T}^{(0)} \cdot \nabla_{h} \boldsymbol{u}_{T}^{(0)}\right\rangle-\left\langle\overline{\boldsymbol{u}_{T}^{(0)} \cdot \nabla_{h} \boldsymbol{u}_{T}^{(0)}}\right\rangle, \\
& \frac{\partial w_{w}^{(1)}}{\partial t_{0}}+\frac{\partial p_{w}^{(1)}}{\partial z}=b_{w}^{(1)},
\end{aligned}
$$




$$
\begin{aligned}
& \frac{\partial b_{w}^{(1)}}{\partial t_{0}}+w_{w}^{(1)}=-\boldsymbol{u}_{T}^{(0)} \cdot \nabla_{h} b_{e}^{(0)}+\overline{\boldsymbol{u}_{T}^{(0)} \cdot \nabla_{h} b_{e}^{(0)}}, \\
& \frac{\partial u_{w}^{(1)}}{\partial x}+\frac{\partial v_{w}^{(1)}}{\partial y}+\frac{\partial w_{w}^{(1)}}{\partial z}=0,
\end{aligned}
$$

which can be combined and expressed as a forced wave equation,

$$
\frac{\partial^{2}}{\partial t_{0}^{2}}\left(\frac{\partial^{2} w_{w}^{(1)}}{\partial z^{2}}\right)+\nabla^{2} w_{w}^{(1)}=\sum_{i=1}^{3} F_{i}^{(0)},
$$

with

$$
\begin{aligned}
& F_{1}^{(0)}=\frac{\partial^{2}}{\partial t_{0} \partial z} \nabla_{h} \cdot\left[\left(\boldsymbol{u}_{h}^{(0)} \cdot \nabla_{h}\right) \boldsymbol{u}_{h}^{(0)}\right], \\
& F_{2}^{(0)}=-\nabla_{h}^{2}\left[\boldsymbol{u}_{h}^{(0)} \cdot \nabla_{h} b^{(0)}\right], \\
& \left.F_{3}^{(0)}=\frac{\partial}{\partial z}\left[\boldsymbol{e}_{3} \cdot\left[\nabla \times\left(\boldsymbol{u}^{0)} \cdot \nabla\right) \boldsymbol{u}_{h}^{(0)}\right)\right]\right],
\end{aligned}
$$

where the $F_{i}^{(0)}$ notation indicates dependence on the lowest-order solutions. Terms with factors of $w^{(0)}$ do not contribute at this order, since $w^{(0)} \equiv 0$. Generally, the solution can be expressed in terms of a Green's function integral.

\subsection{Example}

To illustrate the theory, we consider simple, unimodal initial conditions consisting of three-dimensional Taylor-Green vortices. In non-dimensional form,

$$
\begin{aligned}
& u_{e}(t=0)=\cos k_{1} x \sin l_{1} y \cos m_{1} z, \\
& v_{e}(t=0)=-\frac{k_{1}}{l_{1}} \sin k_{1} x \cos l_{1} y \cos m_{1} z, \\
& w_{e}(t=0)=0, \\
& b_{e}(t=0)=\frac{m_{1}}{l_{1}} \cos k_{1} x \cos l_{1} y \sin m_{1} z, \\
& p_{e}(t=0)=\frac{1}{l_{2}} \cos k_{1} x \cos l_{1} y \cos m_{1} z .
\end{aligned}
$$

Taylor-Green vortices constitute an exact stationary solution of the unforced, inviscid, nonlinear equations of motion. More general, surface-intensified representations of the eddy field will be examined in the numerical simulations.

The eddy field solution is

$$
\begin{aligned}
& u_{e}^{(0)}=U_{e}\left(t_{1}\right) \cos k_{1} x \sin l_{1} y \cos m_{1} z, \\
& v_{e}^{(0)}=-\frac{U_{e}\left(t_{1}\right) k_{1}}{l_{1}} \sin k_{1} x \cos l_{1} y \cos m_{1} z \\
& b_{e}^{(0)}=\frac{U_{e}\left(t_{1}\right) m_{1}}{l} \cos k_{1} x \cos l_{1} y \sin m_{1} z \\
& p_{e}^{(0)}=\frac{U_{e}\left(t_{1}\right)}{l_{1}} \cos k_{1} x \cos l_{1} y \cos m_{1} z
\end{aligned}
$$


where $U_{e}(0)=1$. Lowest-order eddy solutions do not deviate from their initial state on the fast time scale. Slow-time behaviour remains undetermined at this order.

The barotropic tide solution was derived in the previous section and will not be repeated. Moreover, as in the general case, no waves are excited at $O(1)$.

Since nonlinear eddy-eddy terms are zero, the slow-time behaviour of the eddy field reduces to

$$
\frac{\partial \Pi^{(0)}}{\partial t_{1}}=0
$$

We now focus on the $O(\epsilon)$ wave equation,

$$
\frac{\partial^{2}}{\partial t_{0}^{2}}\left(\frac{\partial^{2} w^{(1)}}{\partial z^{2}}\right)+\nabla_{h}^{2} w^{(1)}+\frac{\partial^{2} w^{(1)}}{\partial z^{2}}=F_{1}^{(0)}+F_{2}^{(0)}+F_{3}^{(0)} .
$$

Each forcing term $F_{i}^{(0)}$ contains sinusoidal contributions from eddy-eddy, tide-eddy and tide-tide interactions. For the purpose of our study, it is sufficient to restrict attention to eddy-tide contributions, since these are the only ones that can combine spatial and temporal scales required for projection onto the internal wave band. Ford, McIntyre \& Norton (2000) demonstrated this requirement in the context of shallowwater wave-vortex interactions. Eddy-tide nonlinear terms are of the form $\sin \psi$ and $\cos \psi$, where the phase $\psi$ is

$$
\psi=\left[k_{1} x \pm\left(l_{0} \pm l_{1}\right) y \pm m_{1} z \pm \omega_{0} t_{0}\right]
$$

The $\cos \psi$ and $\sin \psi$ terms do not generally represent freely propagating waves unless eddy and tidal spatial scales satisfy the dispersion relation, here given in dimensional form as

$$
\omega_{0}^{2}=f^{2}+N^{2} \frac{k_{1}^{2}+\left(l_{0} \pm l_{1}\right)^{2}}{m_{1}^{2}} .
$$

In this case (3.27) is forced resonantly with similar response to that of a harmonic oscillator forced at its natural frequency. Under forced resonant conditions, solutions to (3.27) behave as $t_{0} \sin \psi$ and $t_{0} \cos \psi$.

Substituting $l_{0}, k_{1}, l_{1}$ and $m_{1}$ into (3.29), introducing $\theta$ as the angle between the eddy wavevector $\kappa_{1}$ and the horizontal plane, and assuming circular eddies,

$$
k_{1}=l_{1}=\frac{\sqrt{2}}{2} \kappa_{1} \cos \theta,
$$

yields an expression for (normalized) resonant $l_{0}$ as a function of $\theta$,

$$
\frac{l_{0}}{\left\|\kappa_{1}\right\|}= \pm \frac{\sqrt{2}}{2} \cos \theta \pm \sqrt{\left(\frac{\omega_{0}^{2}}{N^{2}}-\frac{f^{2}}{N^{2}}\right) \sin ^{2} \theta-\frac{1}{2} \cos ^{2} \theta}
$$

The locus of resonant $l_{0}$ values is shown in figure 2 . In the ocean, geostrophic eddies are characterized by $m_{1} / k_{1} \gg 1$, corresponding to values of $\theta \approx \pi / 2$ and small values of $l_{0}$. As we shall see in the next paragraph, tide-eddy interaction coefficients vanish identically when $l_{0}=0$, but physically relevant resonances can occur when $l_{0} /\left\|\kappa_{1}\right\|$ is small, albeit non-zero. 


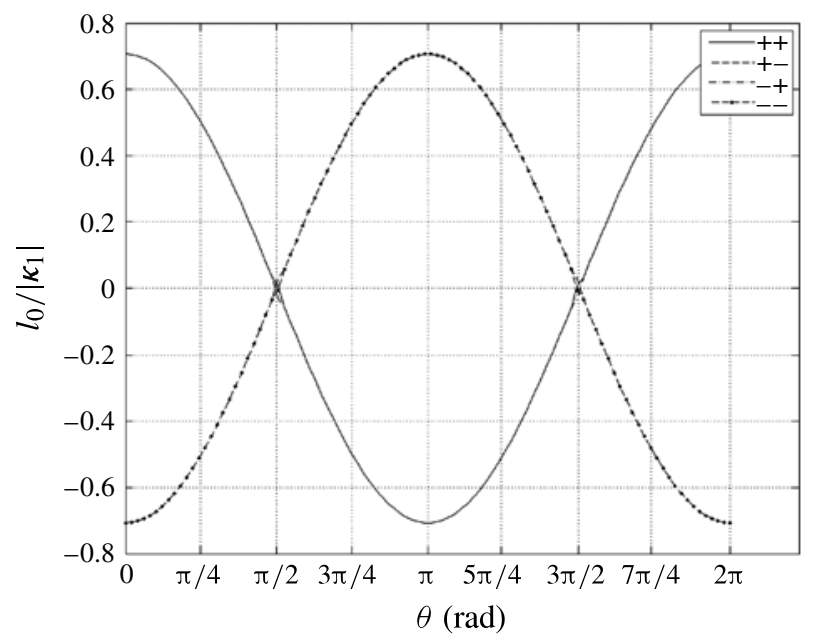

FIGURE 2. Locus of resonant tidal wavenumbers $l_{0}$ (normalized by $\left\|\boldsymbol{\kappa}_{1}\right\|$ ) as a function of $\theta$ where $\tan \theta=m_{1} / \sqrt{\left(k_{1}^{2}+l_{1}^{2}\right)}, f=10^{-4} \mathrm{~s}^{-1}$ and $N=5 \times 10^{-3} \mathrm{~s}^{-1}$. The four branches refer to the \pm branches of (3.31). The ++ and -- branches overlap, as do the +- and -+ branches. Therefore, only two curves can be distinguished.

The coefficients of $\sin \psi$ and $\cos \psi$ on the right-hand side of (3.27) may be combined and written succinctly as

$$
\begin{aligned}
& S^{ \pm}\left(t_{1}\right)=u_{e}^{(0)}\left(t_{1}\right) u_{T}^{(0)}\left(t_{1}\right) \frac{k_{1} l_{0} m_{1}}{8}\left( \pm 1 \pm \frac{l_{0}}{l_{1}}\right), \\
& C^{ \pm}\left(t_{1}\right)= \pm u_{e}^{(0)}\left(t_{1}\right) u_{T}^{(0)}\left(t_{1}\right) \frac{k_{1} l_{0} m_{1}}{8 \omega_{0}},
\end{aligned}
$$

where $S^{ \pm}$and $C^{ \pm}$denote sine and cosine, coefficients respectively. Resonant solutions to (3.27) are written as

$$
w^{(1)}=\frac{S^{ \pm}\left(t_{1}\right)}{2 m_{1}^{2} \omega_{0}} t_{0} \sin \psi+\frac{C^{ \pm}\left(t_{1}\right)}{2 m_{1}^{2} \omega_{0}} t_{0} \cos \psi .
$$

Again assuming $k_{1}=l_{1}, C^{ \pm}$and $S^{ \pm}$achieve extrema when $l_{0}=0$ and $l_{0}=$ $\pm l_{1} / 2$. The former corresponds to a minimum (zero forcing) and the latter to maximum or minimum pairs associated with wavenumbers $\left\{k_{1}, \pm\left(l_{0}+l_{1}\right), \pm m_{1}\right\}$ or $\left\{k_{1}, \pm\left(l_{0}-l_{1}\right), \pm m_{1}\right\}$. While different wavenumber signs represent different directions of phase propagation, they do not affect the frequency of the associated waves. Therefore, if resonant conditions are met for, say, $\left\{k_{1}, l_{0}+l_{1}, m_{1}\right\}$, they will also hold for $\left\{k_{1},-\left(l_{0}+l_{1}\right), m_{1}\right\},\left\{k_{1}, l_{0}+l_{1},-m_{1}\right\}$ and $\left\{k_{1},-\left(l_{0}+l_{1}\right),-m_{1}\right\}$.

When barotropic tidal wavenumber $l_{0}=0$, then

$$
\nabla_{h} \cdot \boldsymbol{u}_{e}^{(0)}=0 \quad \Longrightarrow \quad F_{1}^{(0)}=0,
$$

and from thermal-wind balance,

$$
F_{2}^{(0)}=-F_{3}^{(0)} \Longrightarrow F_{2}^{(0)}+F_{3}^{(0)}=0 .
$$

Therefore, if the barotropic tide velocity is uniform, no waves can be excited since tide-eddy forcing terms are zero, independently of whether resonance conditions are 
satisfied or not. Physically, this situation corresponds to a periodic advection of the entire water column by the tidal current. Without any horizontal modulation, this type of motion cannot transfer horizontal energy to vertical motions.

For the purpose of our study, it is not necessary to pursue the analysis further. The technique above has provided a formal framework for delineating eddy from barotropic tide and baroclinic waves. Beyond yielding low-order solutions to the eddy-tide interaction problem, the weakly nonlinear multiple-scale analysis has been useful in identifying eddy and barotropic tidal scales for which resonance is possible. When resonant conditions are met, excited wave velocities will grow as $O(\epsilon t)$. These solutions remain valid up to $t \sim 1 / \epsilon$, at which time the wave velocity may become $O$ (1) compared to the barotropic tide velocities. In principle, the analysis should be carried out to the next order of approximation to ensure the boundedness of solutions and the self-consistency of the asymptotic expansions. This would entail taking into account terms of $O(\gamma)$ and would require considerable additional effort. Moreover, carrying out solutions to higher order would not be particularly useful since the theory presented here is ultimately limited by the absence of dissipation. Without dissipation, tidal forcing will eventually lead to the unphysical situation of unbounded energy growth.

In the following section, a set of numerical simulations designed to validate and complement the weakly nonlinear theory is presented.

\section{Numerical simulations}

The pseudo-spectral numerical model solves the three-dimensional Boussinesq equations on the $f$ plane (Winters $\&$ de la Fuente 2012) in a domain of dimensions $L_{x} \times L_{y} \times L_{z}=160 \mathrm{~km} \times 40 \mathrm{~km} \times 2400 \mathrm{~m}$. Boundary conditions are periodic in both horizontal directions and free-slip in the vertical. Numerical stability is maintained with a sixth-order hyperviscous operator $D$, designed to damp the smallest resolved scales at the same rate in each direction. In spectral space,

$$
D(k, l, m)=-v_{6}\left\{\left(k / k_{\max }\right)^{6}+\left(l / l_{\max }\right)^{6}+\left(m / m_{\max }\right)^{6}\right\},
$$

where $v_{6}$ is the hyperviscous coefficient, and $k_{\max }, l_{\max }$ and $m_{\max }$ denote maximum wavenumbers in the $x, y$ and $z$ directions, respectively. The same operator is used in the density equation, with a hyperdiffusion coefficient $\kappa_{6}$ replacing $v_{6}$. In all cases, $v_{6}=\kappa_{6}=7.8 \times 10^{-5} \mathrm{~s}^{-1}$, which is the minimum value required to prevent the onset of numerical instability.

To assess the role of the barotropic tide, we compare the evolution of four simulations, runs $\mathrm{A}, \mathrm{B}, \mathrm{C}$ and $\mathrm{D}$. In runs $\mathrm{A}-\mathrm{C}$, the initial condition consists of an eddy field in geostrophic and hydrostatic balance. In order to facilitate visualization of internal wave generation, the eddy field is confined to the upper and middle regions of the domain (figure 3). The eddy field differs slightly from the one used in the theoretical analysis. In the horizontal, the array of Taylor-Green vortices is modulated by

$$
R(x)=\mathrm{e}^{-\beta\left(L_{x} / 2-x\right)^{2}},
$$

and in the vertical by

$$
S(z)=\mathrm{e}^{-\alpha\left(L_{z}-z\right)} \cos m_{1} z
$$

where $\alpha=6 / L_{z}$ and $\beta=\left(12 / L_{x}\right)^{2}$. Restricting the spatial extent of the eddy field facilitates visualization of radiating internal waves. 

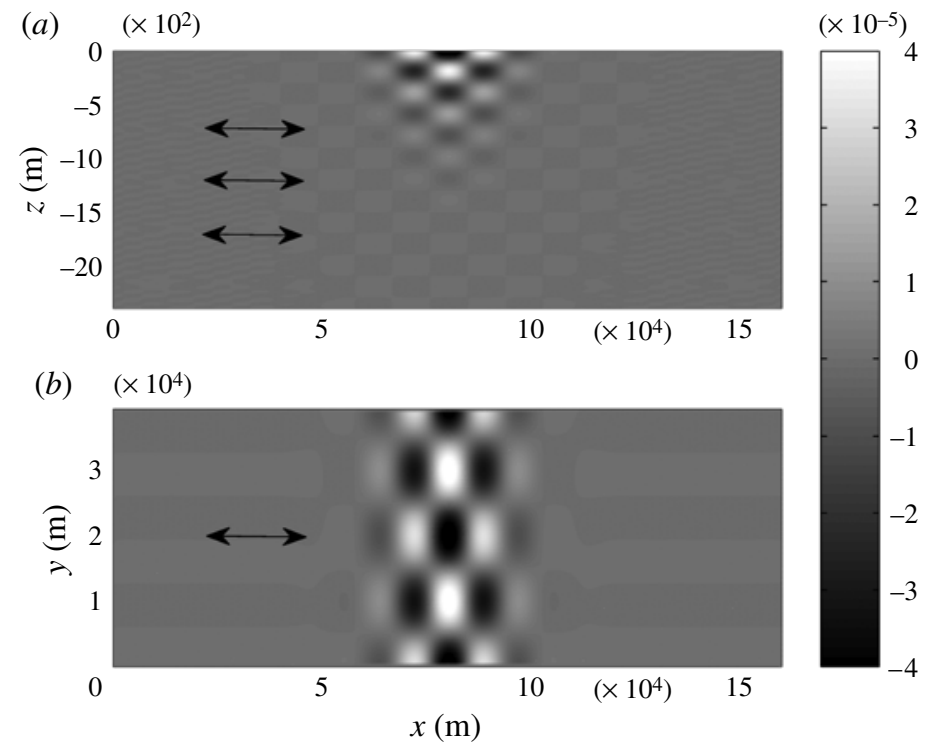

FIGURE 3. (a) Vertical and $(b)$ horizontal cross-sections of vorticity field at $t=0$. Arrows indicate the direction of the barotropic tidal forcing in runs $\mathrm{B}$ and $\mathrm{C}$.

The balanced initial conditions are

$$
\begin{aligned}
u_{e} & =\frac{U R(x) S(z)}{l_{1}} \cos k_{1} x \sin l_{1} y, \\
v_{e} & =U S(z) \cos l_{1} y\left[R^{\prime}(x) \cos k_{1} x-k_{1} R(x) \sin k_{1} x\right], \\
w_{e} & =0 \\
\rho_{e} & =-\frac{U f \rho_{0}}{g l_{1}} S^{\prime}(z) R(x) \cos k_{1} x \cos l_{1} y
\end{aligned}
$$

and $U=0.1 \mathrm{~m} \mathrm{~s}^{-1}$.

The eddy field in run $\mathrm{D}$ includes a broad range of eddy scales, is confined to the upper ocean as in runs $\mathrm{B}$ and $\mathrm{C}$, but extends over the entire horizontal domain. As in the theory, barotropic tidal forcing is introduced as a body force. To ensure smooth solutions, the tide is ramped up to full strength over five tidal cycles with a hyperbolic tangent. Coriolis and buoyancy frequencies are $10^{-4} \mathrm{~s}^{-1}$ and $5 \times 10^{-3} \mathrm{~s}^{-1}$, respectively. Thus, $N / f=50$, which is typical of the mid-latitude pycnocline.

Run A, with tidal forcing turned off, provides a base case for wave radiation resulting from eddy field imbalances only. Runs $\mathrm{B}$ and $\mathrm{C}$ differ in the choice of horizontal tidal scale $\left(\lambda_{0}=2 \pi / l_{0}\right)$. In run $\mathrm{B}, \lambda_{0}$ is much larger than the scale of the dominant eddies, as is characteristic of the deep ocean. Run $\mathrm{C}$ has $\lambda_{0}$ comparable to the eddy scale, as might occur on the continental shelf, and resonant conditions are satisfied. The same tidal forcing as in run $\mathrm{C}$ is applied to run $\mathrm{D}$, but the eddy field in this simulation contains a broad range of scales. In this case, $\lambda_{1}=2 \pi / l_{1}$ represents the dominant eddy scale. Run D will enable us to assess whether this type of resonant response is likely to occur in a general eddy field that has not a priori been tuned for resonance. 
For all cases discussed below, the Rossby number is defined as $\epsilon=2 \pi U / f L$ (note the factor of $2 \pi$, which was omitted in the definition in $\S 2.2$ ). Based on initial eddy velocity $U=0.1 \mathrm{~m} \mathrm{~s}^{-1}$ and radius $L=20 \mathrm{~km}$, we have $\epsilon=0.3$. This corresponds to a slow time scale of the order of three inertial periods, or five tidal periods.

Equations (3.2) and (3.5) are used to extract tide and eddy components. For the eddy field, temporal averaging is performed with a running mean. As defined in (3.2), $T$ is two inertial periods, approximately equidistant from fast and slow times. Horizontal residual velocities $u_{r}$ and $v_{r}$ are obtained by subtracting tide and eddy contributions from the total horizontal velocity. When resonant excitation occurs, we expect that residual velocities will be dominated by a linear internal wave, and $u_{r}, v_{r}$ and $w$ should satisfy linear polarization relations,

$$
\begin{aligned}
& u_{r}=A \cos \phi, \\
& v_{r}=\frac{\left(f^{2}+\omega_{0}^{2}\right) k_{2} l_{2}+\mathrm{i} f \omega_{1}\left(k_{2}^{2}-l_{2}^{2}\right)}{f^{2} l_{2}^{2}+\omega_{0}^{2} k_{2}^{2}} u_{r}, \\
& w=-\frac{\left(k_{2} u_{r}+l_{2} v_{r}\right)}{m_{2}},
\end{aligned}
$$

where $\phi=k_{2} x+l_{2} y+m_{2} z-\omega_{0} t, k_{2}=k_{1}, l_{2}=\left(l_{0} \pm l_{1}\right)$ and $m_{2}=m_{1}$. Equations (4.5a-c) will be used in analysing runs A-D to diagnose whether inertia-gravity waves are being excited.

\subsection{Run A: base run, no tidal forcing}

All four runs, including the unforced run A, exhibit some low-level internal wave oscillations. In run $\mathrm{A}$, weak radiation occurs at the onset of the simulation because the degree of balance of the initial eddy field is inherently limited by finite numerical resolution. This slight imbalance triggers the geostrophic adjustment of the Taylor-Green vortices, which results in radiation of a weak internal wave field. The dependence on resolution was confirmed by noting a decrease in initial wave radiation with increasing numerical resolution (not shown). Another source of waves arises because the array of localized vortices is not an exact solution of the nonlinear equations (in contrast to the periodic array of Taylor-Green vortices used in $\S 3$ ). Consequently, over many eddy time scales, nonlinear effects act to distort the initial state, resulting in wave radiation. This was verified numerically by performing a linear simulation in which nonlinear terms were set to zero. In this scenario, the vortices retained their initial balanced state and the resulting residual signal dropped significantly compared to the nonlinear case.

However, neither mechanism discussed above provides a significant wave source. This is confirmed by the temporal evolution of vertically averaged and residual velocities at a fixed point away from the eddy field (figure 4). Large spikes in the vertical velocity occur sporadically, but do not grow discernibly. Horizontal residual velocities display some degree of polarization and increase slightly between 15 and 20 tidal periods in response to eddy field nonlinear distortions.

\subsection{Run B: uniform tidal forcing}

Tidal forcing is spatially constant in run $\mathrm{B}$, corresponding to the theoretical case $l_{0}=0$. The tidal flow spins up quickly; $u_{T}$ and $v_{T}$ have comparable amplitudes (figure $5 a$ ), and are $\pi / 2$ out of phase, as predicted by the theory $(3.13 a, b)$. Residual horizontal velocities display organized and coherent behaviour, oscillate at the tidal frequency and are larger than in run A (figure $5 b$ ). However, the vertical velocity is incoherent, 

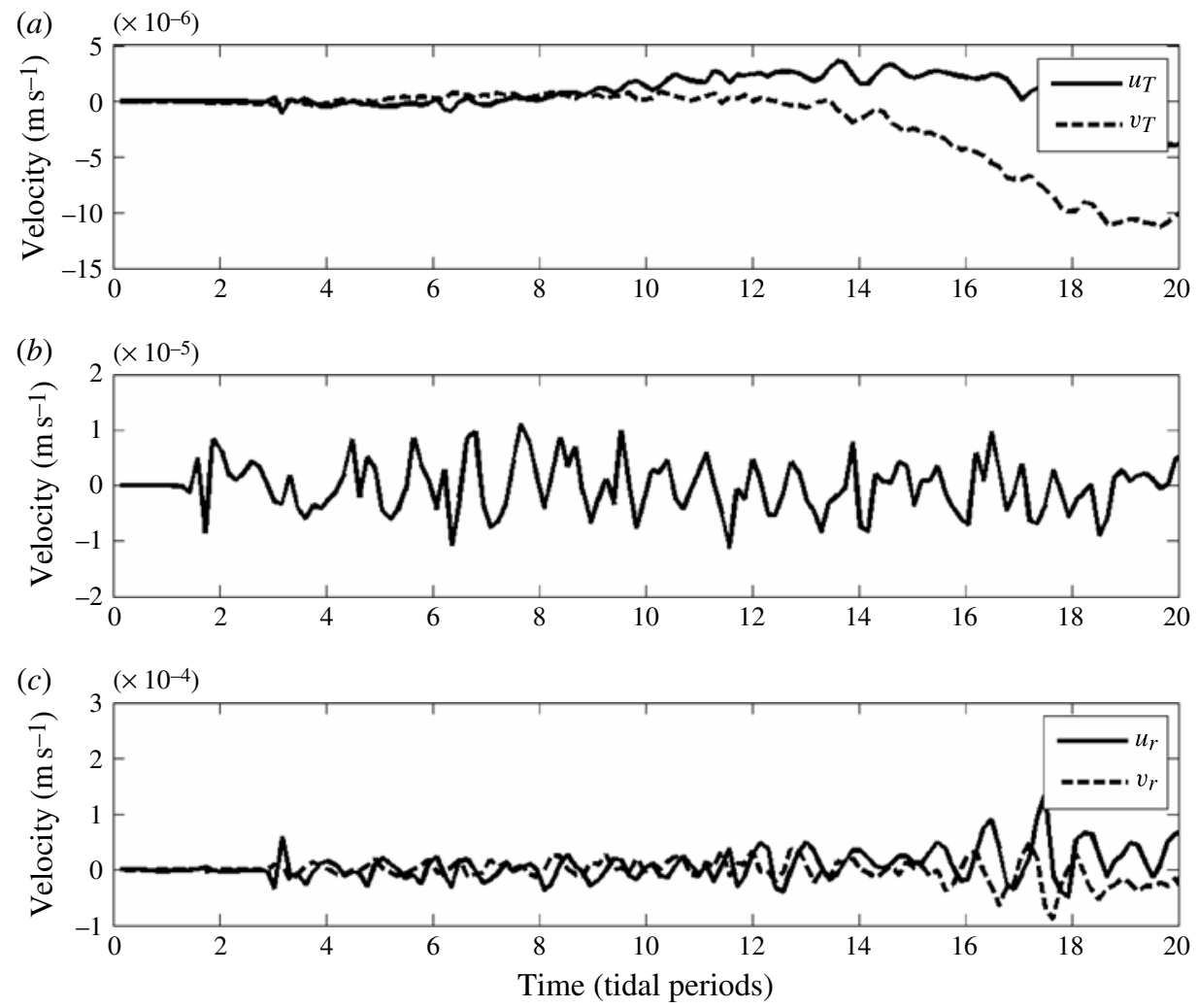

FIGURE 4. Run A (unforced): time evolution of $(a)$ vertically averaged tidal velocities, (b) vertical velocity and $(c)$ residual horizontal velocities $u_{r}$ and $v_{r}$. All velocities are recorded at a fixed point at mid-depth and away from the eddy field region.

with residual horizontal velocities, and oscillates more rapidly. This suggests that the residual tidal ellipses are not propagating vertically according to polarization relations (4.5a). Maximum residual amplitudes do not exceed $10^{-4} \mathrm{~m} \mathrm{~s}^{-1}$.

\subsection{Run C: resonant tidal forcing}

Prior to settling on the particular set of parameters chosen for run $\mathrm{C}$, several simulations were performed with resonant parameters at or close to the maximum forcing $l_{0}=2 l_{1}$ predicted by the theory. No discernible differences in the intensity of the wave field could be detected. Therefore, we chose the set of resonant wavenumbers that produced the cleanest resonant response. In this simulation, eddy and tidal scales satisfy the resonance conditions given in $\S 2.1$, with $l_{0} /\left\|\kappa_{1}\right\|=0.02$ (figure 2) and with tidal and eddy scales $L_{y}=3 \lambda_{0}=2 \lambda_{1}$. To allow for horizontal and vertical propagation of generated waves, $L_{x}=4 L_{y}$ and $L_{z}=6 \times\left(2 \pi / m_{1}\right)$. Oceanic eddies are typically deeper than the ones modelled in our study, but, given the constraints of limited computational resolution, parameters were chosen to facilitate visualization of radiating internal waves away from the region occupied by eddies. Moreover, a comparison with theory cannot involve eddies with a strong barotropic signature, which would project onto the tidal mode and invalidate the flow decomposition. 

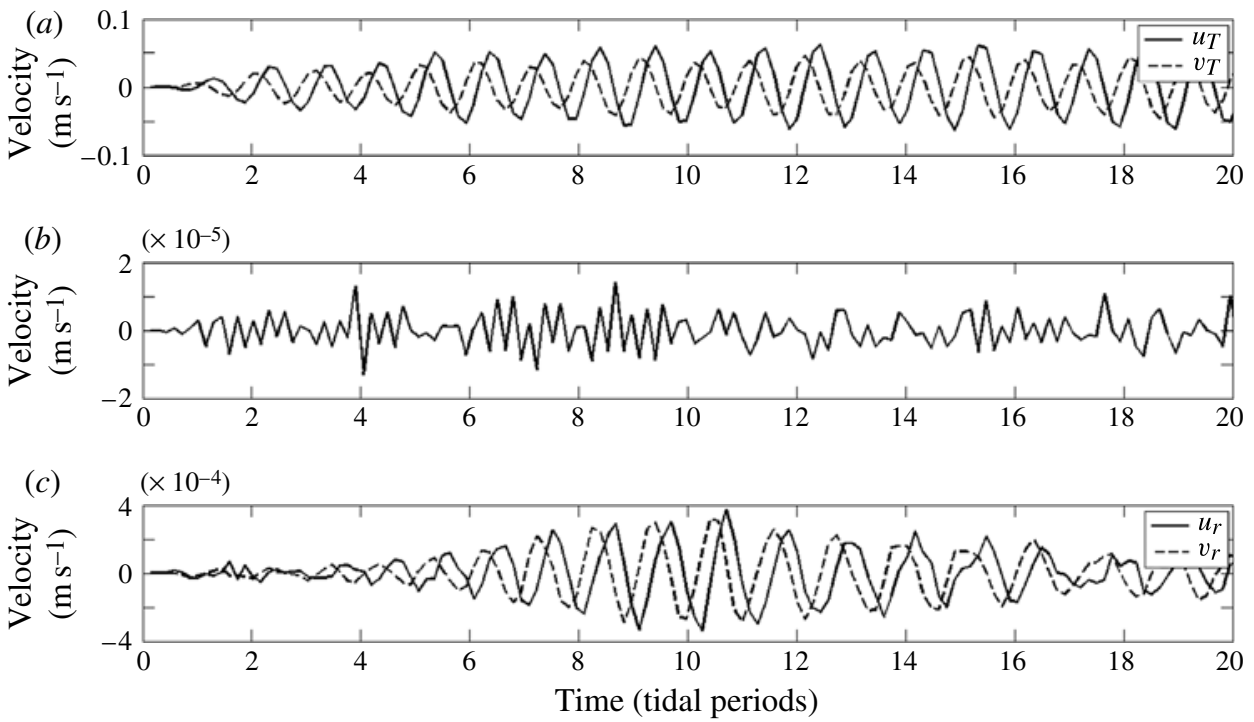

FIGURE 5. Run B (uniform tidal forcing): time evolution of (a) vertically averaged tidal velocities, $(b)$ vertical velocity and $(c)$ residual horizontal velocities $u_{r}$ and $v_{r}$. All velocities are recorded at a fixed point at mid-depth and away from the central eddy region.

Cross-sections of the vertical velocity are shown in figure 6. At early times, $w$ exhibits little variation in $y$ or $z$ as evidenced by the signal at the edges of the domain in figure $6(a, b)$. At later times, the signal develops mode 1 and mode 6 structures in $y$ and $z$, respectively (figure $6 c, f$ ).

Hovmöller diagrams overlaid with theoretical phase speeds (figure 7) confirm that the emitted waves are travelling with vertical phase speed $\omega_{0} / m_{1}$. In the horizontal, the negative phase speed matches the theoretical value of $-\omega_{0} / k_{1}$. The waves with positive phase speed, on the other hand, exhibit a phase speed of $0.5 \omega_{0} / k_{1}$. The asymmetry in the emitted waves is also evident in figure $6(b, c)$, where the wave signal in the negative $x$ direction is noticeably stronger than in the positive $x$ direction. The differences between leftward and rightward emitted waves can be reconciled with the corresponding asymmetry in the relative vertical vorticity (figure 8). Kunze (1985) showed that strong geostrophic vorticity $\zeta$ modulates the internal wave dispersion relation $k^{2}+l^{2}=\left(\omega^{2}-(f+\zeta / 2)^{2}\right) m^{2}$. Here, $k$ is probably being modulated in opposite directions by the ambient vorticity field. Tide, vertical and residual velocity components are shown in figure 9. Relations (4.5) are used to confirm that the developing signal in figure $6(b, c)$ has frequency $\omega_{0}$ and wavevector $\left\{k_{1},\left(l_{0}-l_{1}\right), m_{1}\right\}$, as predicted by theory. The wave amplitude grows on the slow time scale, reaching its peak value as a coherent signal in 10-12 tidal periods. It may seem puzzling that, even when resonant conditions are met, the vertical velocity remains $O(\epsilon)$ compared to eddy and barotropic tide velocities. This can be attributed to the fact that the scaling employed in the non-dimensionalization $(\$ 2.3)$ imposes the constraint that the ratio of vertical to horizontal scales is $O(\epsilon)$. Therefore, an additional implicit factor of $\epsilon$ is present and the effective forcing amplitude is $O\left(\epsilon^{2}\right)$.

The linear wave signal persists until $t=10$ (tidal periods), then becomes decorrelated at later times, presumably as a result of interactions with re-entrant waves at the boundaries of the periodic domain. Another noteworthy observation is the 

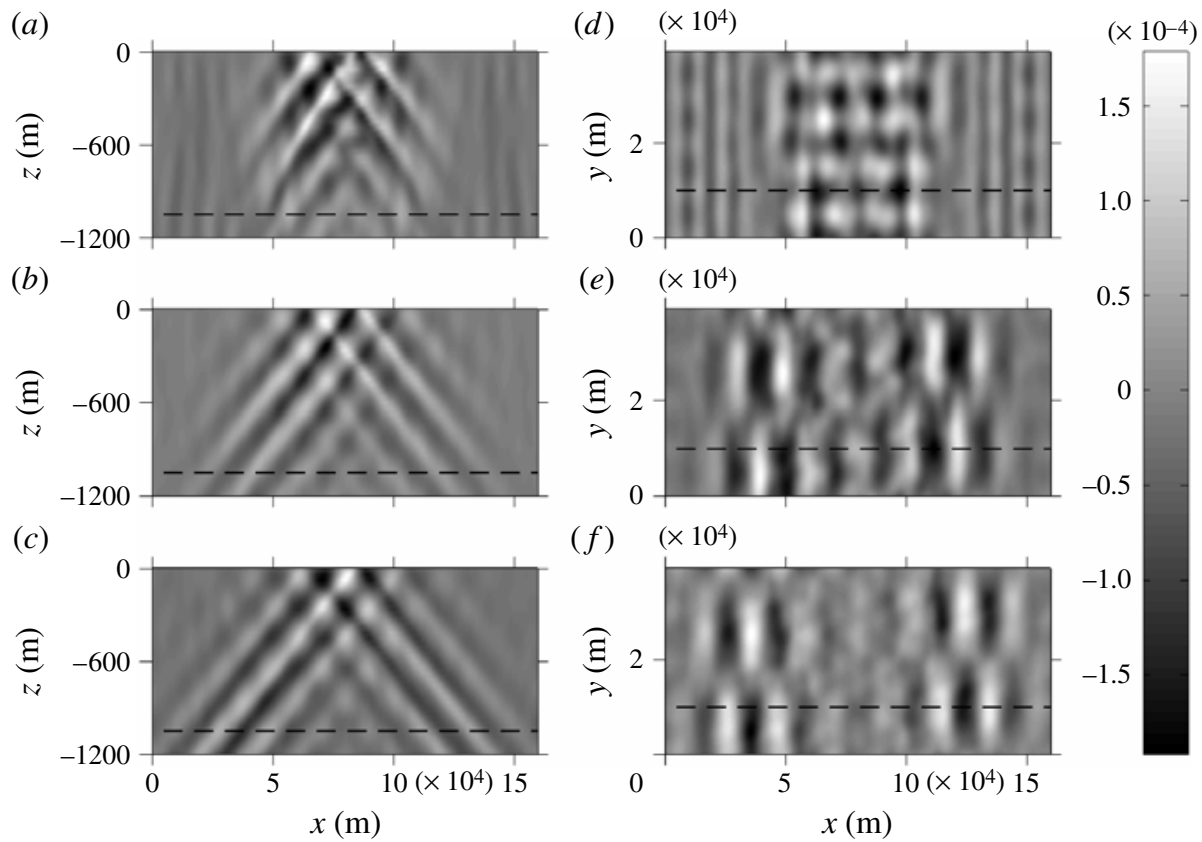

FIgURE 6. Run C: cross-sections of $w$ at $(a, d) t=3.5 T,(b, e) t=7 T$ and $(c, f) 10 T$, where $T$ is the tidal period. Panels $(a, b, c)$ represent vertical cross-sections (only top half of domain is shown); panels $(d, e, f)$ are horizontal cross-sections. The dashed lines indicate corresponding positions of horizontal and vertical planes. Displayed data span $\pm 2 \times 10^{-4} \mathrm{~m} \mathrm{~s}^{-1}$.
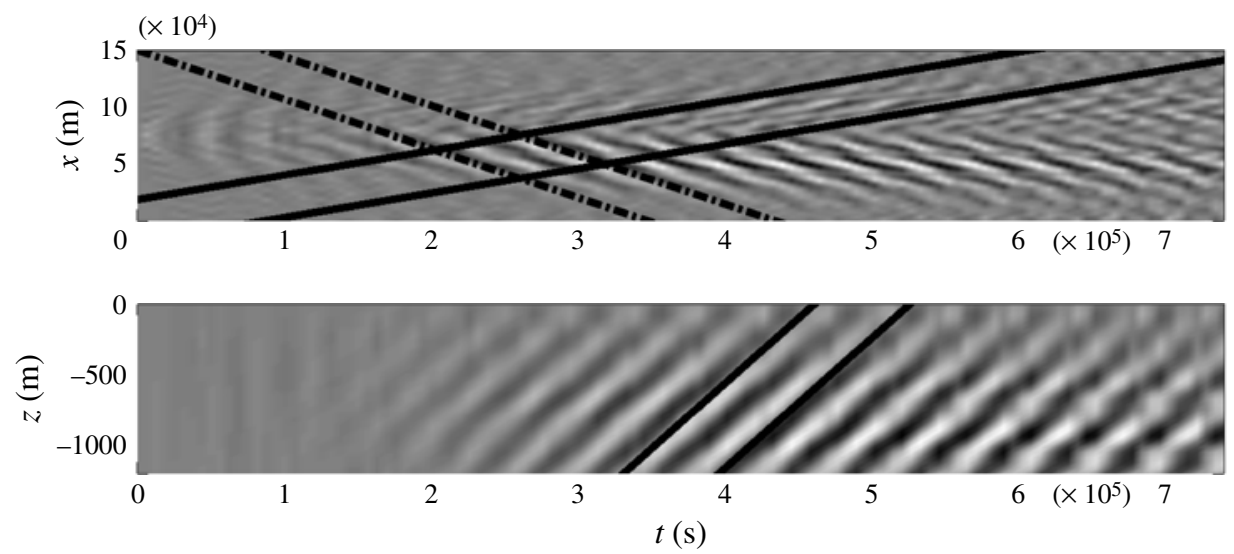

FIGURE 7. Run C: $(a)$ horizontal and $(b)$ vertical Hovmöller plots. Solid and dash-dotted lines represent theoretical phase speeds. Only the upper half of the vertical domain is shown. Vertical and horizontal plane positions are indicated by the dashed lines on figure 6 .

absence of a tidal ellipse: in contrast to run $\mathrm{B}$, where tidal $u_{T}$ and $v_{T}$ components are in linear balance and elliptically polarized, $v_{T}$ in run $\mathrm{C}$ is negligible and the amplitude of $u_{T}$ is smaller than in run B by roughly a factor of two. This behaviour is consistent with the theory: (i) when $l_{0} \neq 0, v_{T}$ must be zero in order for the flow 


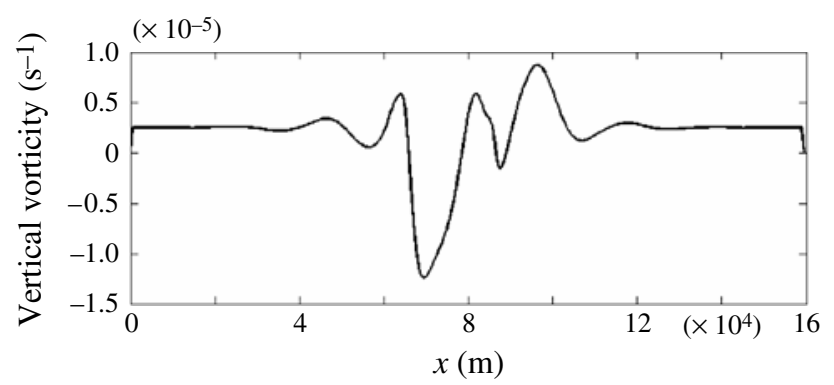

FIGURE 8 . Run C: vertical vorticity at $t=7 T$, at the surface. The $y$ position is indicated by the dashed line on figure $6(d, e, f)$. The non-zero signal in the far field is an artefact of the initialization.
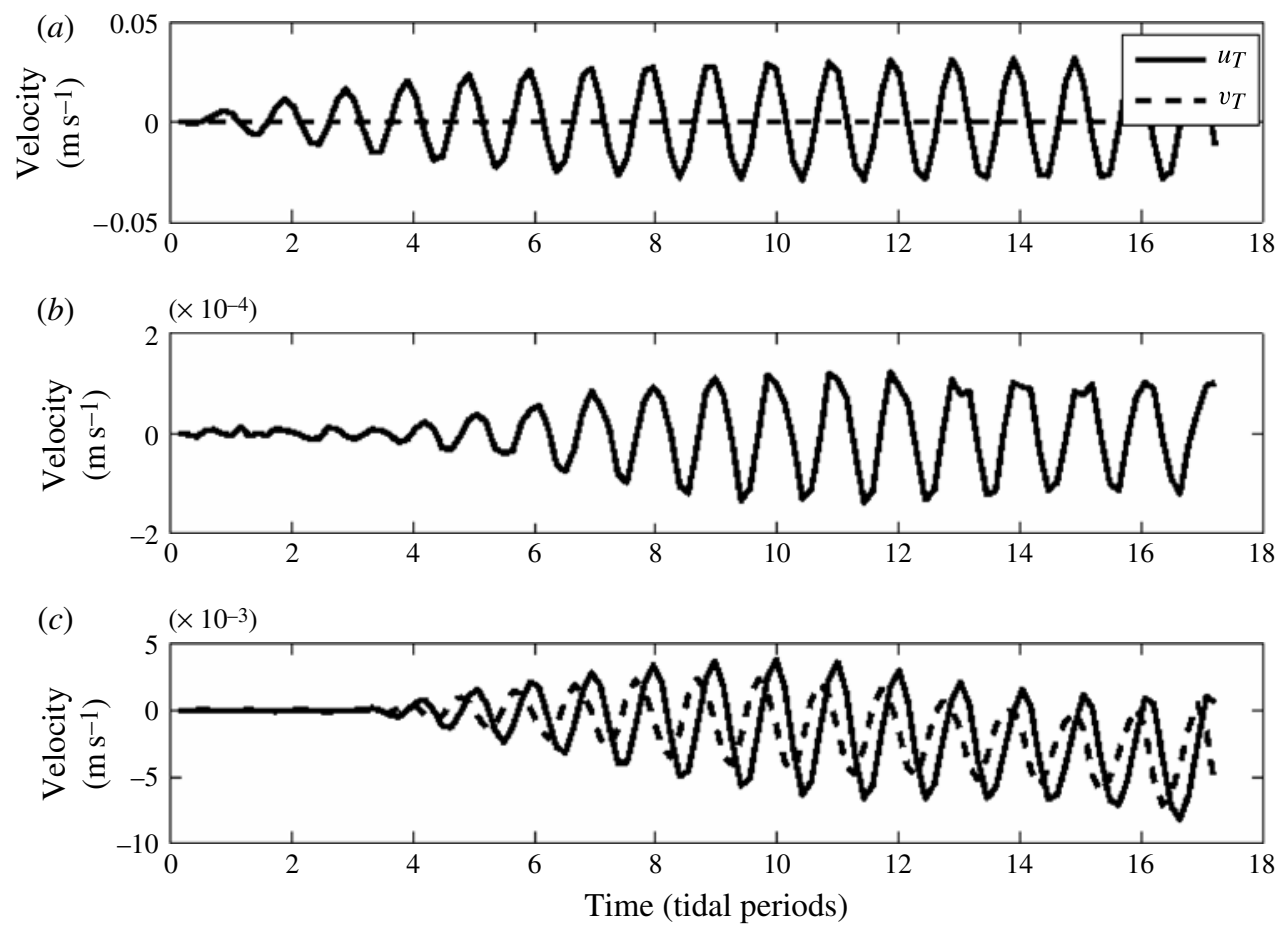

FIGURE 9. Run C (tidal forcing modulated on eddy spatial scale): time evolution of (a) vertically averaged tidal velocities, $(b)$ vertical velocity and (c) residual horizontal velocities $u_{r}$ and $v_{r}$. All velocities are recorded at a fixed point at mid-depth and away from the central eddy region.

to satisfy continuity; and (ii) a comparison of (3.10) and (3.14) confirms that the amplitude of $u_{T}^{(0)}$ is reduced by a factor of $1 / \omega_{0}^{2}$ when $l_{0} \neq 0$. In non-dimensional form, $\omega_{0}=1.45 \Rightarrow 1 / \omega_{0}^{2}=0.47 \approx 0.5$.

Tidal, eddy and residual energies are shown in figure 10. The following discussion focuses on the time interval between 5 and 15 tidal periods. The ramp-up period during which the flow is adjusting to tidal forcing is excluded because it exhibits 

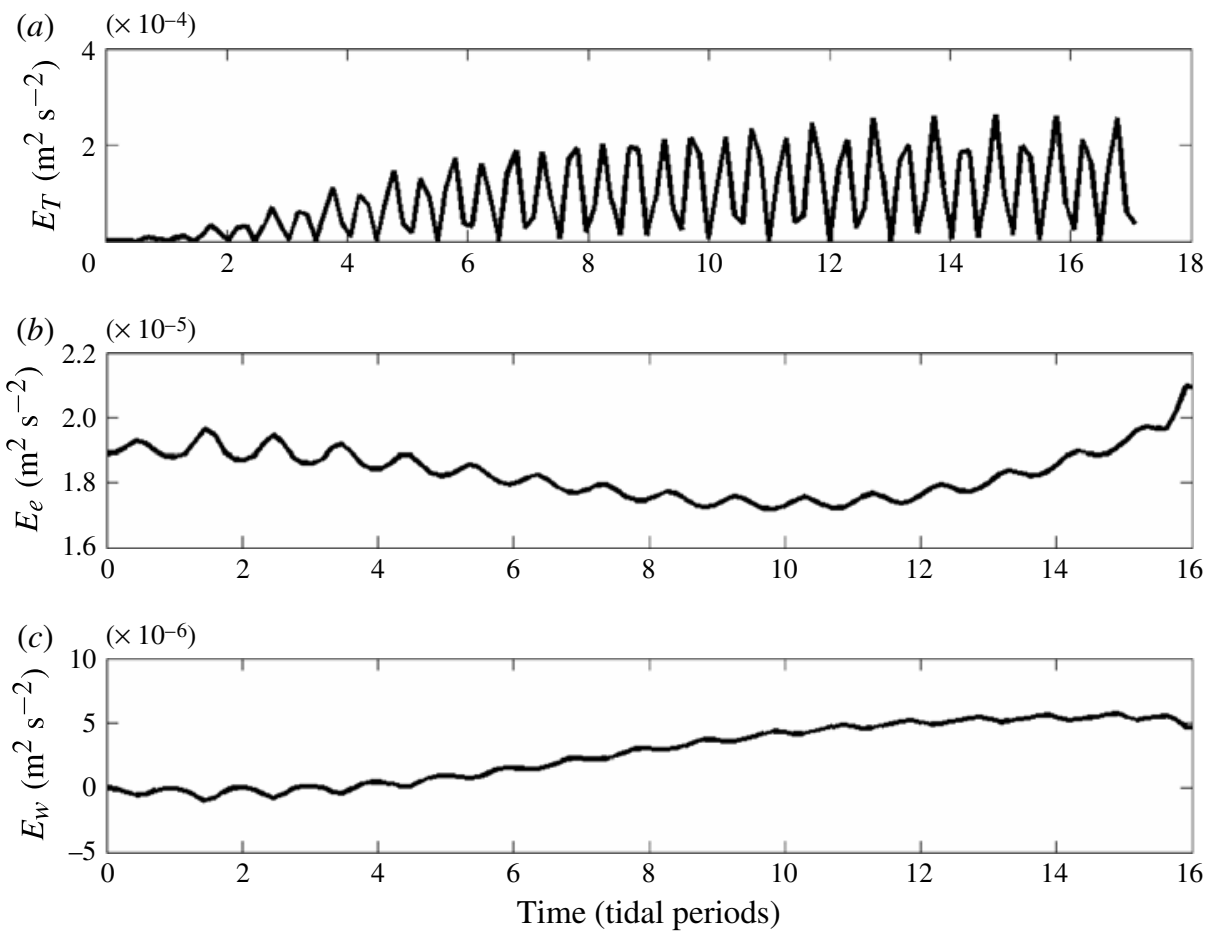

FIGURE 10. Volume-averaged energies (kinetic plus available potential) for $(a)$ barotropic tide, $(b)$ eddy field and $(c)$ wave field for run $\mathrm{C}$. The large fluctuations in barotropic tidal energy in panel $(a)$ are due to modulation by the forcing.

unphysical behaviour, e.g. negative residual energy (figure 10b). Once the tidal signal has been established, tidal energy accounts on average for $75 \%$ of the total energy, with the eddy field energy roughly $20 \%$ and the residual wave energy about $5 \%$ of the total energy. Tidal energy oscillates with a period of $\pi / \omega_{0}$, whereas the eddy energy modulates over a longer period. As seen in figure 10(b), eddy energy is not entirely devoid of fast oscillations. This apparent discrepancy between theoretical and numerical results is explained by the fact that the theory assumes that $\epsilon$ is infinitely small and that fast and slow time scales do not overlap. In our simulations, however, $\epsilon$ is small but finite. Residual and eddy energies remain in phase throughout the simulation and out of phase with the tidal energy, confirming that the tide supplies the energy for the excited waves. Additional confirmation that the residual energy represents waves is provided by examining the ratio of residual kinetic $\left(K E_{r}\right)$ to potential $\left(P E_{r}\right)$ energies. For inertia-gravity waves (Gill 1982), one has

$$
r=\frac{\left\langle K E_{r}\right\rangle}{\left\langle P E_{r}\right\rangle}=\frac{\omega^{2}+f^{2}}{\omega^{2}-f^{2}} .
$$

For our parameters, the computed value of $r=2.5$ compares well with the theoretical value of 2.7. This resonant interaction results in a stable periodic exchange of energy between tide and the eddy/wave components.

We also find good agreement between theoretical $w$, given by (3.34), and numerically computed $w$ for the time frame over which the fast-time solution is 


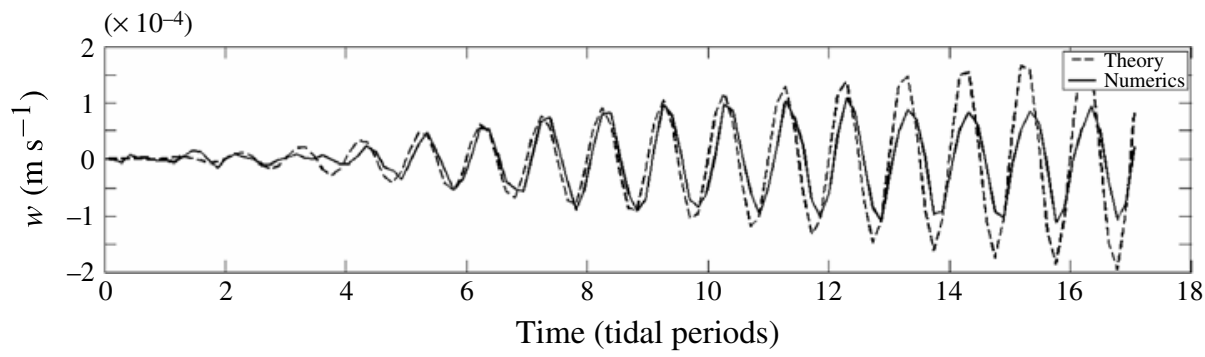

FIGURE 11. Comparison of theoretical (dashed) and numerical (solid) $w$. The theoretical solution has been multiplied by the same ramping function used in the numerical model.

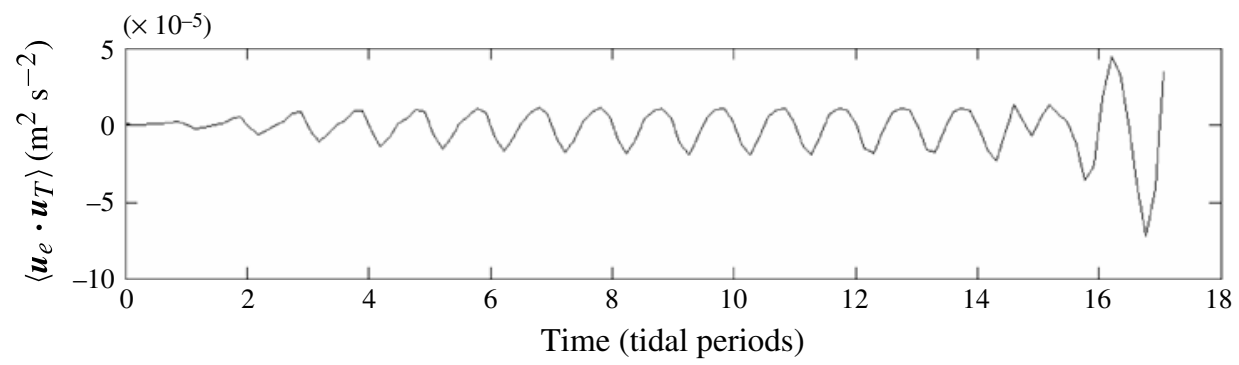

FIGURE 12. Volume-averaged cross-term $\boldsymbol{u}_{e} \cdot \boldsymbol{u}_{T}$ in run C.

valid, i.e. for times less than $1 / \epsilon$ (figure 11). Capturing the slow-time behaviour of the $O(\epsilon)$ solution would presumably yield agreement over longer times, but this is beyond the scope of our study. Moreover, the theory presented here does not take into account dissipative effects and will, therefore, become invalid for long times.

Finally, we verify that the tide-eddy decomposition is valid. In order for this flow decomposition to be useful, we must have

$$
\frac{1}{2}\left\|\boldsymbol{u}_{T}+\boldsymbol{u}_{e}\right\|^{2} \approx \frac{1}{2}\left(\left\|\boldsymbol{u}_{T}\right\|^{2}+\left\|\boldsymbol{u}_{e}\right\|^{2}\right)
$$

implying that

$$
\boldsymbol{u}_{T} \cdot \boldsymbol{u}_{e} \ll \frac{1}{2}\left(\left\|\boldsymbol{u}_{T}\right\|^{2}+\left\|\boldsymbol{u}_{e}\right\|^{2}\right) .
$$

The left-hand side of (4.8) is plotted in figure 12. The cross-term oscillates about zero and remains three orders of magnitude less than the tidal energy and one order of magnitude below the eddy energy over the duration of the simulation, confirming that $\boldsymbol{u}_{T}$ and $\boldsymbol{u}_{e}$ have negligible projections onto one another. This validates the utility of the flow decomposition in analysing the simulations.

\subsection{Run D: tidal forcing of a more general eddy field}

Run D considers the impact of tidal forcing on an eddy field containing a range of spatial scales. The initial condition is constructed by seeding a field of regularly distributed Taylor-Green vortices throughout the entire domain with a randomly phased higher-mode perturbation, and allowing it to evolve until a statistically quasistationary state is achieved. For ease of interpretation, the unperturbed Taylor-Green vortices prior to spin-up have the same spatial scales and are confined vertically as 

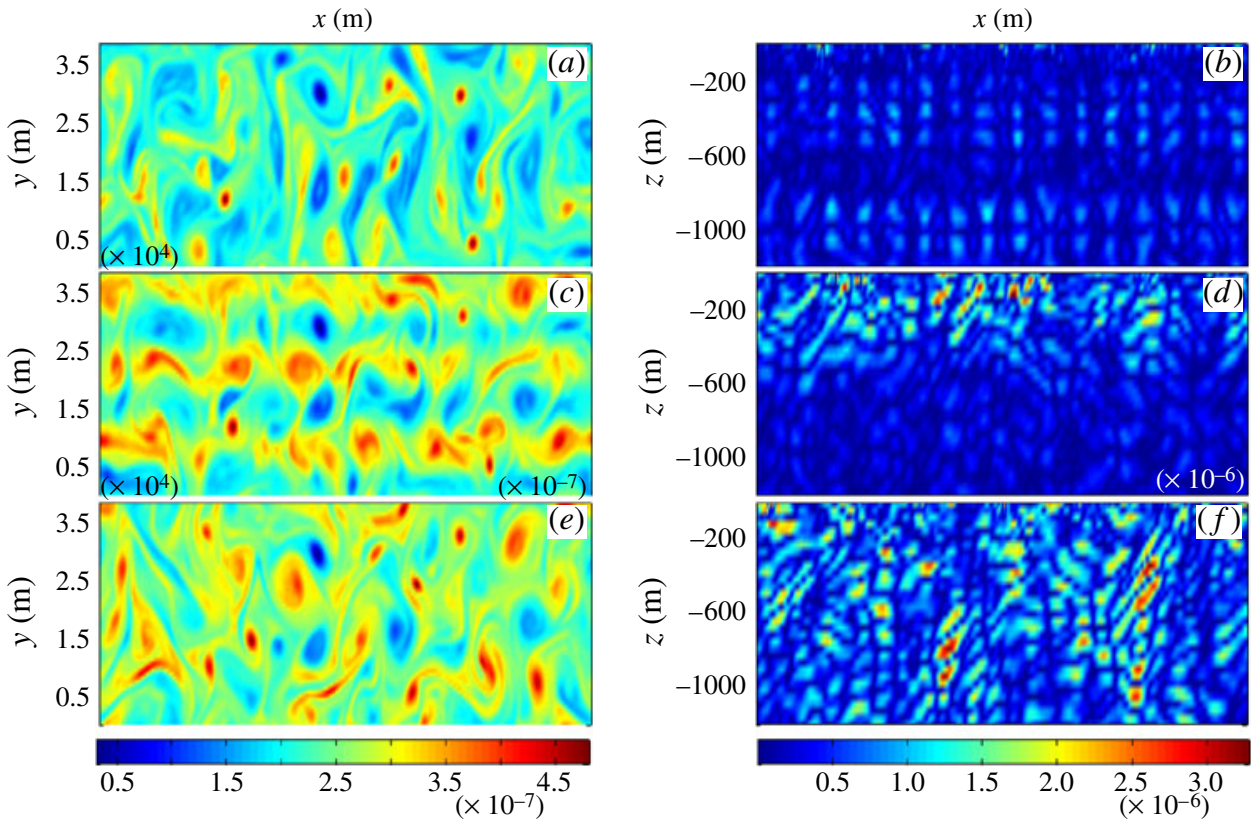

FIGURE 13. Run D: $(a, c, e)$ plan views of potential vorticity and $(b, d, f)$ vertical crosssections of horizontal divergence magnitude. Panels represent the flow $(a, b)$ at the onset of tidal forcing $(t=0),(c, d)$ at $t=5 T$ and $(e, f)$ at $t=10 T$. Only the top half of the domain is shown in vertical cross-sections $(b),(d)$ and $(f)$. Units on the colour bars are $\mathrm{m}^{-1} \mathrm{~s}^{-1}(P V)$ and $\mathrm{s}^{-1}$ (divergence).

in run C. The initial potential vorticity field thus constructed is shown in figure 13(a). Once the flow has spun up, after about 20T, the same barotropic forcing as in run $\mathrm{C}$ is ramped up over five tidal periods (figure 14a).

In this simulation, the eddy flow has evolved significantly from a regular Taylor-Green array, and considerable merging and straining of vortices has occurred prior to the onset of tidal forcing (figure 13a). Here, potential vorticity $(P V)$,

$$
P V=\frac{\left(\nabla \times \boldsymbol{u}+f \hat{\boldsymbol{\imath}}_{3}\right) \cdot \nabla(\bar{\rho}+\rho)}{\rho_{0}},
$$

which has no signature on the (non-breaking) internal wave field, is used to characterize the eddy field. Horizontal divergence provides a good description of the wave field (figure 13). Evidence of tidal forcing is seen in the striated pattern exhibited by the eddy field at $t=25 T$ (figure 13c). Prior to the onset of tidal forcing, the wave field is weak (figure 13b). By $t=25 T$, wave generation begins (figure $13 d$ ). Over time, wave generation intensifies in the surface layers and wave packets also become visible at depth (figure 13f). Anticyclones deepen and the internal waves tend to concentrate in the confluent strain-dominated regions between eddies (not shown).

As in run $\mathrm{C}$, residual horizontal velocities and vertical velocity exhibit the characteristic behaviour of linear internal waves of frequency $\omega_{0}$ (figure 14). $P V$ exhibits a peak at the tidal forcing scale and two weaker peaks, one corresponding to the original Taylor-Green scale and the other at $k_{x}=1.5 \times 10^{-4} \mathrm{~m}^{-1}$, suggesting that a transfer of $P V$ to larger scales has taken place prior to forcing (figure 15a). At later times, $P V$ is concentrated over a broader range of scales (figure $15 a$ ). Horizontal 

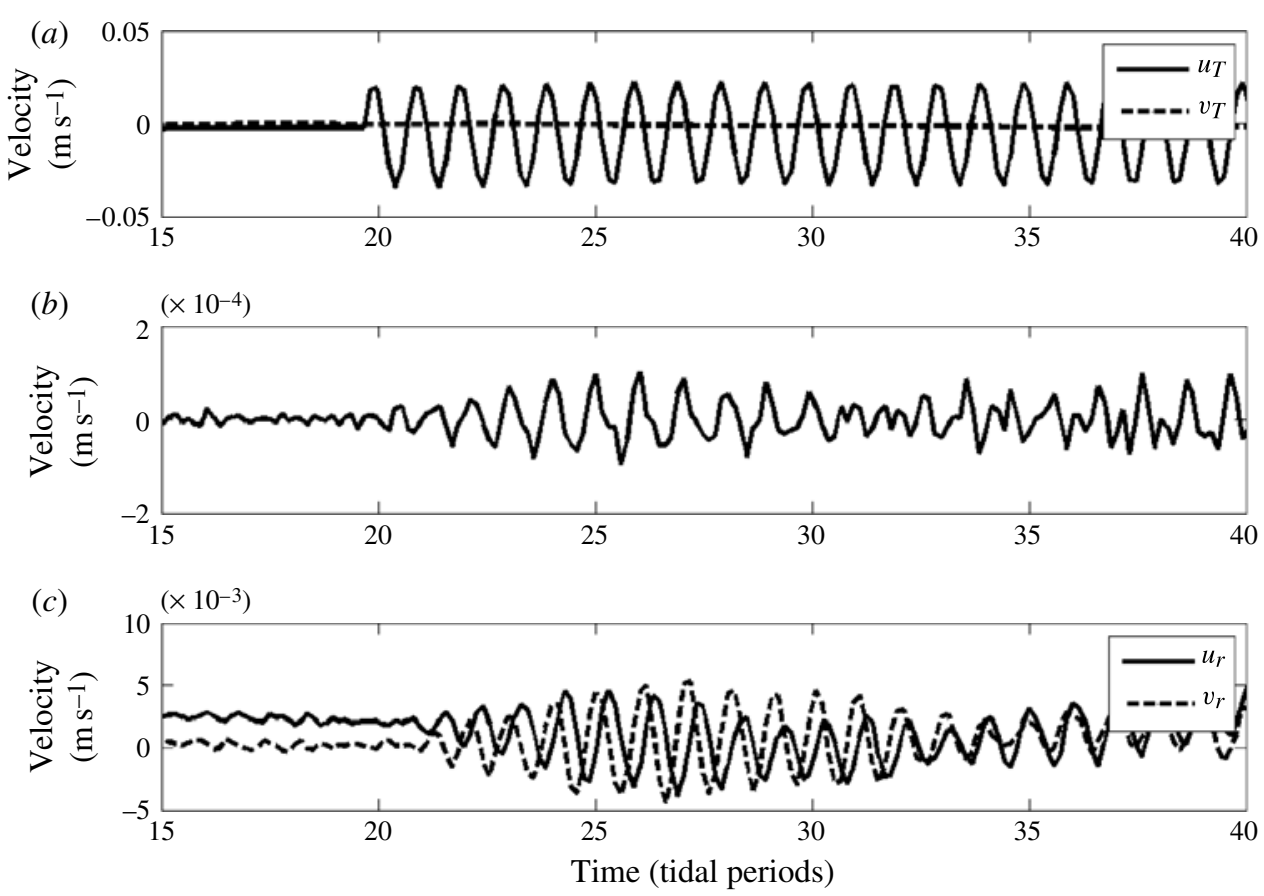

FIGURE 14. Run D (tidal forcing of a general eddy field): time evolution of $(a)$ vertically averaged tidal velocities, $(b)$ vertical velocity and $(c)$ residual horizontal velocities $u_{r}$ and $v_{r}$. Velocities are recorded at a single point, at mid-depth.

divergence (internal wave) levels are small initially. A peak corresponding to $k_{1}$ is visible by $t=6 T$ after the onset of tidal forcing. A cascade to higher harmonics has taken place by $t=10 T$. Potential enstrophy and horizontal divergence variance $y$ spectra are shown in figure 16. The divergence exhibits a peak at $k_{y}=7.8 \times 10^{-4} \mathrm{~m}^{-1}$, which corresponds to mode 5 . In this case, the strongest resonant wave generation is associated with $l_{0}+l_{1}$, in contrast to run $\mathrm{C}$, where $l_{0}-l_{1}$ dominated. This increase in horizontal wavenumber is accompanied by an increase in vertical wavenumber, as evidenced by the shorter vertical wavelength of the propagating waves (figure $13 f$ ).

This final simulation confirms that resonantly excited waves will be preferentially excited in the presence of a broad range of eddy scales, though eddy-tide resonances are not likely to provide a significant source of internal waves, given the requirement that eddy and tidal flows vary on comparable horizontal scales, and this condition is not typically encountered in the ocean.

\section{Conclusions}

We have examined the interaction between barotropic tidal currents and a field of baroclinic geostrophic eddies both theoretically and numerically as a potential mechanism for generating internal gravity waves. We find that the eddy field evolution is governed by the conservation of potential vorticity and is independent of the tide. This behaviour is reminiscent of situations where the eddy field acts as a catalyst in triggering interactions between fast flow components without participating in the energy exchange. 

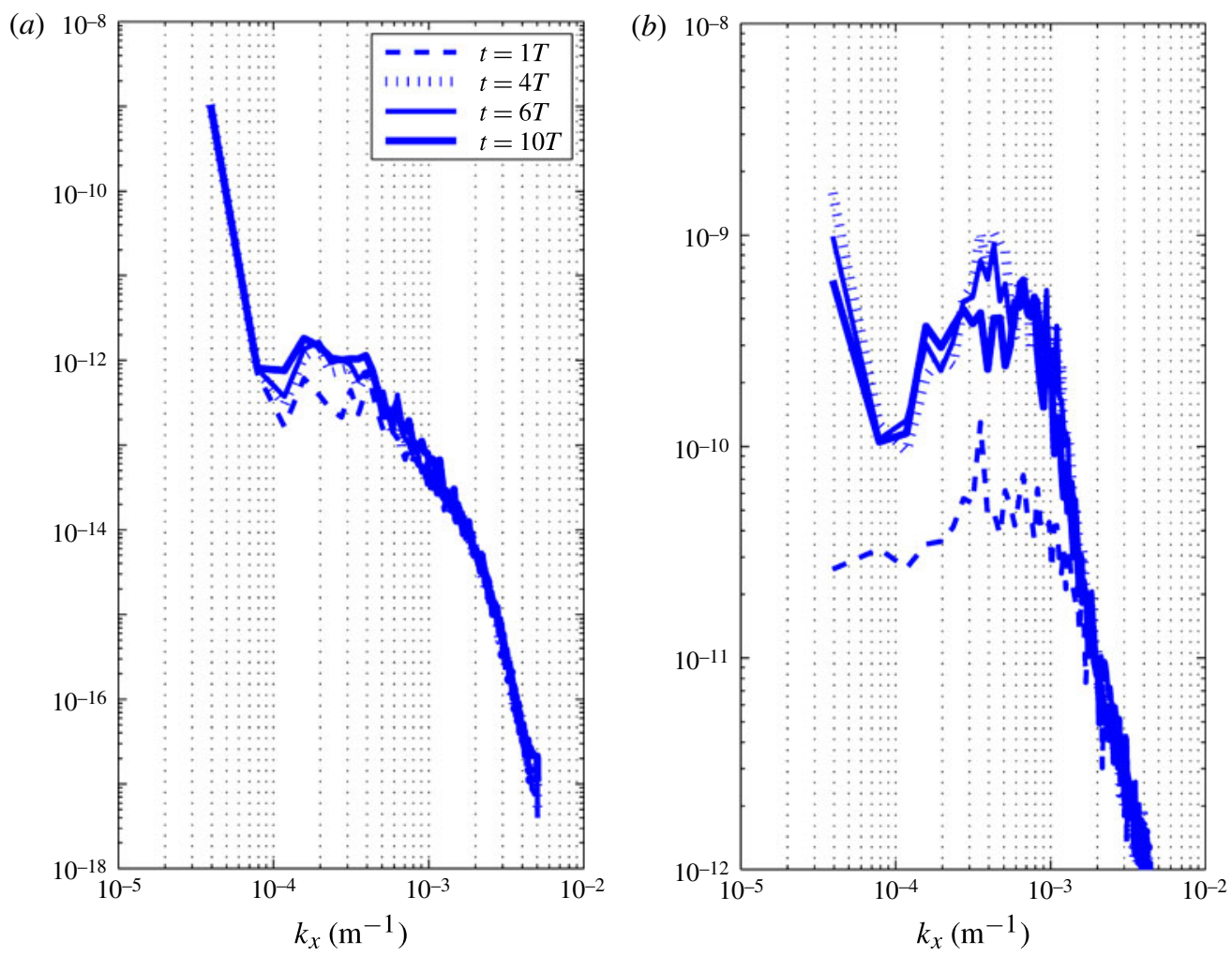

FIgURE 15. (Colour online) Run D: variance spectra of (a) $P V$ and $(b)$ horizontal divergence versus horizontal wavenumber $k_{x}$. Tidal forcing scale is $2 \pi / L_{x}=3.9 \times 10^{-5} \mathrm{~m}^{-1}$ and initial Taylor-Green eddy scale at $k_{2}=3.1 \times 10^{-4} \mathrm{~m}^{-1}$. As in figure $13, t=0$ corresponds to the time at which tidal forcing is initiated.

If the oscillating flow has infinite horizontal wavelength, that is, no convergences, the eddy field is advected back and forth and no energy is transferred to the internal wave band. The largest transfer rates occur if the horizontal wavelengths of the eddies and tidal forcing are of similar scale, with a weak maximum when the tidal scale is twice that of the eddies. This suggests that this mechanism will not be effective at transferring energy from the surface tides to internal waves in the deep ocean because the Rossby radius $\sim O(N H / f) \sim 100 \mathrm{~km}$ is an order of magnitude shorter than the wavelengths of barotropic tides. Thus, the speculation that eddy-diurnal tide interaction could explain the intense near-inertial waves observed in western North Pacific anticyclonic vortices (Rogachev et al. 1992) is disproved, and it is more likely that the observed oscillations were wind-generated.

The ocean circulation does offer larger eddy scales through upscale energy cascades, but the resulting length scales are too large to match those of internal waves with tidal frequency. Atmospheric forcing generates shallow-water waves over a range of frequencies. Higher frequencies and wavelengths could potentially interact with geostrophic eddies, but these have far less energy associated with them than the tides. Finally, eddy and surface tide length scales become more comparable in shallow water, but, even on continental shelves, they remain widely separated. Thus, while this interaction probably plays a role in the ocean, it will be not be very significant 
(a)

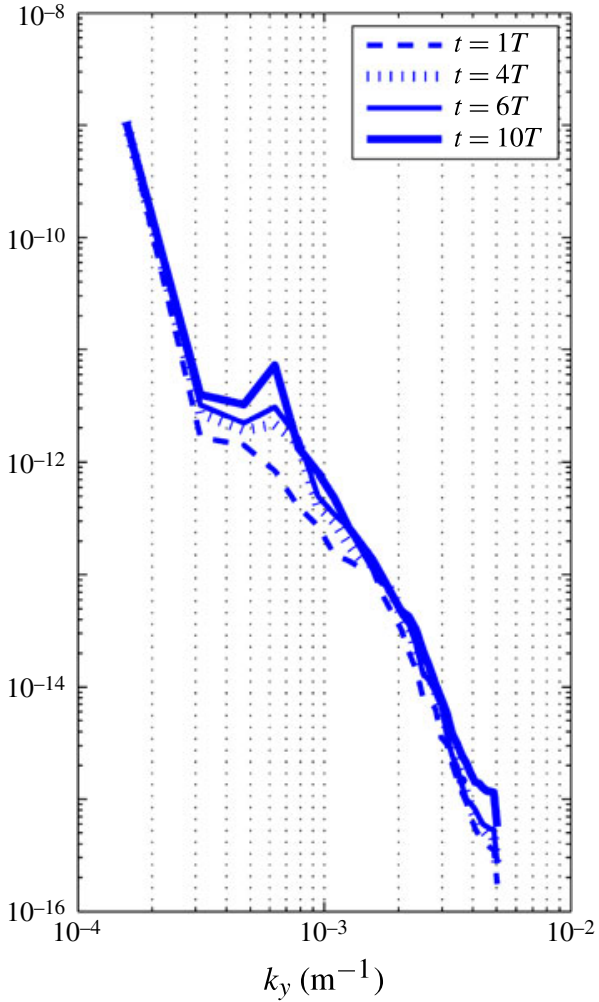

(b)

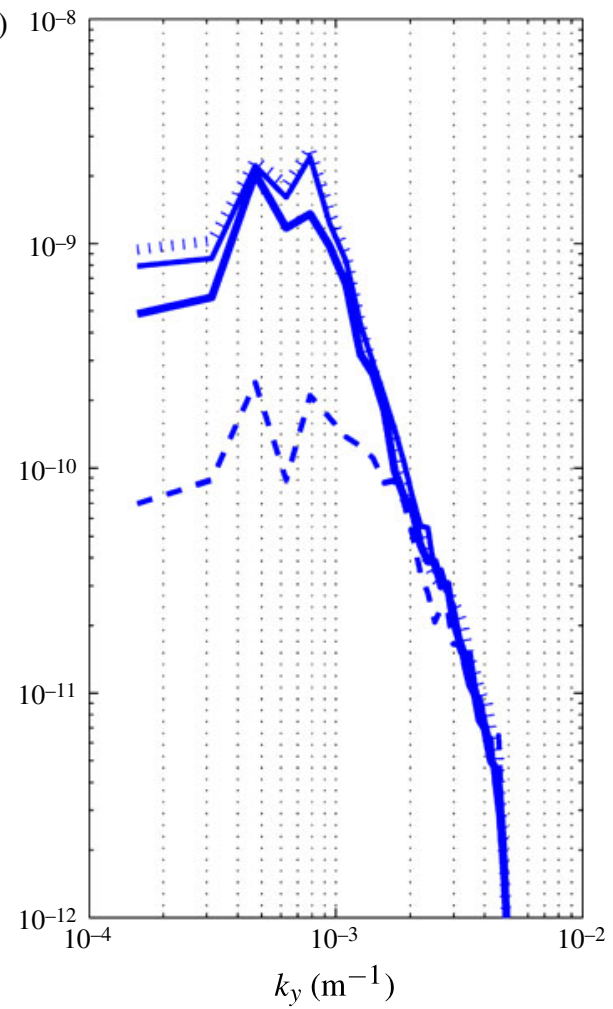

FIgURE 16. (Colour online) Variance spectra of $(a) P V$ and (b) horizontal divergence versus horizontal wavenumber $k_{y}$. Tidal forcing is at $l_{1}=4.7 \times 10^{-4} \mathrm{~m}^{-1}$ and initial Taylor-Green eddy scale at $l_{2}=3.1 \times 10^{-4} \mathrm{~m}^{-1}$. As in figure $13, t=0$ corresponds to the time at which tidal forcing is initiated.

because of a mismatch in the length scales of geostrophic eddies and barotropic tides. Excluded from the present study are cases in which the eddies are themselves forced by wind or other external dynamics. Such forcing could strongly perturb the eddies away from their balanced state and induce a strongly nonlinear response. This scenario corresponds to a fast eddy response and subsequent breakdown in the time scale separation, corresponding to the Rossby number approaching unity. Another possibility for strong wave generation might occur when the horizontal component of rotation cannot be neglected. However, these effects are confined near the turning latitude $\omega \sim f$, where the barotropic tide's horizontal length scales are maximal, and therefore least likely to match eddy field scales. For the same reason, $\beta$-plane considerations are unlikely to allow stronger resonance. These possibilities could ultimately be tested with a different numerical model, but they lie well beyond the scope of the present study.

\section{Acknowledgements}

This work benefited from conversations with J. Toole, D. Schecter and E. Danioux. Insightful suggestions by C. Staquet, V. Zeitlin and two anonymous reviewers greatly improved the manuscript. We thank K. Winters for the use of his numerical code. Nonlinear interaction coefficients were computed with Mathematica. Support for the 
initial phase of this study was provided by the Office of Naval Research, Physical Oceanography Division. Additional support from NSF (OCE-1031286) is gratefully acknowledged.

\section{REFERENCES}

Althaus, A. M., Kunze, E. \& SAnford, T. B. 2003 Internal tide radiation from Mendocino Escarpment. J. Phys. Oceanogr. 33, 1510-1527.

Balmforth, N. J., Ierley, G. R. \& Young, W. R. 2002 Tidal conversion by subcritical topography. J. Phys. Oceanogr. 32, 2900-2914.

BELL, T. H. 1975 Topographically generated internal waves in the open ocean. J. Geophys. Res. 80, 320-327.

Cox, C. \& SAndstrom, H. 1962 Coupling of internal and surface waves in water of variable depth. J. Oceanogr. Soc. Japan 18, 499-513.

DANioux, E. \& KLEIN, P. 2008 A resonance mechanism leading to wind-forced motions with a $2 f$ frequency. J. Phys. Oceanogr. 38 (10), 2322-2329.

Egbert, G. D. \& RAY, R. 2001 Estimates of $M_{2}$ tidal energy dissipation from TOPEX/Poseidon altimeter data. J. Geophys. Res. 106, $22475-22502$.

Ford, R., MCIntyre, M. E. \& Norton, W. A. 2000 Balance and the slow quasimanifold: some explicit results. J. Atmos. Sci. 57 (9), 1236-1254.

Garrett, C. J. R. \& KunZe, E. 2007 Internal tide generation in the deep ocean. Annu. Rev. Fluid Mech. 39, 57-87.

GiLl, A. E. 1982 Atmosphere-Ocean Dynamics. Academic Press.

Holloway, P. E. \& Merrifield, M. A. 1999 Internal tide generation by seamounts, ridges and islands. J. Geophys. Res. 104, 25 937-25951.

Kevorkian, J. \& Cole, J. D. 1981 Perturbation Methods in Applied Mathematics. Springer.

Khatiwala, S. 2003 Generation of internal tides in an ocean of finite depth: analytical and numerical calculation. Deep-Sea Res. 50, 3-21.

KunZe, E. 1985 Near-inertial wave propagation in geostrophic shear. J. Phys. Oceanogr. 15, $544-565$.

Lee, C. M., Kunze, E., Sanford, T. B., Nash, J. D., Merrifield, M. A. \& Holloway, P. E. 2006 Internal tides and turbulence along the 3000-m isobath of the Hawaiian Ridge. J. Phys. Oceanogr. 36, 1165-1183.

LELONG, M.-P. \& RiLEY, J. J. 1991 Internal wave-vortical mode interactions in strongly stratified flows. J. Fluid Mech. 232, 1-19.

Llewellyn Smith, S. G. \& Young, W. R. 2002 Conversion of the barotropic tide. J. Phys. Oceanogr. 32, 1554-1556.

Llewellyn Smith, S. G. \& Young, W. R. 2003 Tidal conversion at a very steep ridge. J. Fluid Mech. 495, 175-191.

MacCready, P. \& PaWlak, G. 2001 Stratified flow along a rough slope: separation drag and wave drag. J. Phys. Oceanogr. 31, 2824-2839.

McComas, C. H. \& BRetherton, F. P. 1977 Resonant interaction of oceanic internal waves. J. Geophys. Res. 83, 1397-1412.

Merrifield, M. A. \& Holloway, P. E. 2002 Model estimates of M2 internal tide energetics at the Hawaiian Ridge. J. Geophys. Res. 107, 3179.

Morozov, E. G. 1995 Semidiurnal internal wave global field. Deep-Sea Res. 42, 135-148.

Müller, P., Holloway, G., Henyey, F. \& Pomphrey, N. 1986 Nonlinear interactions among internal gravity waves. Rev. Geophys. 24, 493-536.

NAsh, J. D., Kunze, E., SAnford, T. B. \& Lee, C. M. 2006 Structure of the baroclinic tide generated at Kaena Ridge, Hawaii. J. Phys. Oceanogr. 36, 1123-1135.

Petrelis, F., Llewellyn Smith, S. G. \& Young, W. R. 2006 Tidal conversion at a submarine ridge. J. Phys. Oceanogr. 36, 1053-1071.

Pingree, R. D., Mardell, G. T. \& New, A. L. 1986 Propagation of internal tides from the upper slopes of the Bay of Biscay. Nature 321, 154-158. 
Pingree, R. D. \& New, A. L. 1989 Downward propagation of internal tide energy into the Bay of Biscay. Deep-Sea Res. 36, 735-758.

RAY, R. D. \& Mitchum, G. T. 1997 Surface manifestation of internal tides in the deep ocean: observations from altimetry and island gauges. Prog. Oceanogr. 40, 135-162.

RezniK, G. M., Zeitlin, V. \& Ben Jelloul, M. 2001 Nonlinear theory of geostrophic adjustment. Part 1. Rotating shallow-water model. J. Fluid Mech. 445, 93-120.

Rogachev, K. A. \& CARMACK, E. C. 2002 Evidence for the trapping and amplification of near-inertial motions in a large anticyclonic ring in the Oyashio Current. J. Oceanogr. 58, 673-682.

Rogachev, K. A., Carmack, E., Miyaki, M., Thompson, R. \& Yurasov, G. I. 1992 Drifting buoy in an anticyclonic eddy of the Oyashio Current. Dokl. Ross. Akad. Nauk 326, 547-550.

Rogachev, K. A., Salomatin, A. S., Yusupov, V. I. \& Carmack, E. C. 1996 On the internal structure of the Kuril Current anticyclonic eddies. Okeanologiya 36, 247-354.

Rudnick, D. L., Boyd, T., Brainard, R. E., Carter, G. S., Egbert, G. D., Gregg, M. C., Holloway, P. E., Klymak, J., Kunze, E., Lee, C. M., Levine, M. D., Luther, D. S., Martin, J., Merrifield, M. A., Nash, J. N., Pinkel, R., Rainville, L. \& SAnford, T. B. 2003 From tides to mixing along the Hawaiian Ridge. Science 301, 355-357.

Simmons, H., Hallberg, R. W. \& Arbic, B. K. 2004 Internal wave generation in a global baroclinic tide model. Deep-Sea Res. 51, 3043-3068.

St Laurent, L. C. \& Garrett, C. J. R. 2002 The role of internal tides in mixing the deep ocean. J. Phys. Oceanogr. 32, 2882-2899.

St Laurent, L. C., Stringer, S., Garrett, C. J. R. \& Perrault-Joncas, D. 2003 The generation of internal tides at abrupt topography. Deep-Sea Res. 50, 987-1003.

THORPE, S. A. 1992 The generation of internal waves by flow over the rough topography of a continental slope. Proc. R. Soc. Lond. A 439A, 115-130.

Vlasenko, V., Stashchuk, N. \& Hutter, K. 2005 Baroclinic Tides: Theoretical Modeling and Observational Evidence. Cambridge University Press.

Waite, M. L. \& BARTEllo, P. 2004 Stratified turbulence dominated by vortical motion. J. Fluid Mech. 517, 281-308.

Winters, K. B. \& DE LA Fuente, A. 2012 Modelling rotating stratified flows at laboratory-scale using spectrally-based DNS. Ocean Model. 49, 47-59.

Wunsch, C. 1975 Internal tides in the ocean. Rev. Geophys. Space Phys. 13, 167-182.

Zeitlin, V., ReZnik, G. M. \& Ben Jelloul, M. 2003 Nonlinear theory of geostrophic adjustment. Part 2. Two-layer and continuously stratified primitive equations. J. Fluid Mech. 491, 207-228. 